\title{
Genome-Wide Association Study and Transcriptome Analysis Reveal Key Genes Affecting Root Growth Dynamics In Rapeseed
}

Keqi Li ( $\sim$ likeqi1218@sina.com)

Oil Crops Research Institute Chinese Academy of Agricultural Sciences https://orcid.org/0000-00027606-2940

\section{Jie Wang}

Oil Crops Research Institute Chinese Academy of Agricultural Sciences

\section{Lieqiong Kuang}

Oil Crops Research Institute Chinese Academy of Agricultural Sciences

\section{Ze Tian}

Oil Crops Research Institute Chinese Academy of Agricultural Sciences

\section{Xinfa Wang}

Oil Crops Research Institute Chinese Academy of Agricultural Sciences

\section{Xiaoling Dun}

Oil Crops Research Institute Chinese Academy of Agricultural Sciences

\section{Jinxing Tu}

Huazhong Agricultural University: Huazhong Agriculture University

Hanzhong Wang

Oil Crops Research Institute Chinese Academy of Agricultural Sciences

\section{Research Article}

Keywords: Brassica napus, root growth, persistent, stage-specific, GWAS, WGCNA

Posted Date: April 14th, 2021

DOI: https://doi.org/10.21203/rs.3.rs-408440/v1

License: (c) (i) This work is licensed under a Creative Commons Attribution 4.0 International License.

Read Full License 
1 Genome-wide association study and transcriptome analysis reveal key genes affecting root 2 growth dynamics in rapeseed

3

4 Keqi $\mathrm{Li}^{1,2,{ }^{\dagger}}$, Jie Wang ${ }^{1{ }^{\dagger}}$, Lieqiong Kuang${ }^{1}$, Ze Tian${ }^{1}$, Xinfa Wang${ }^{1}$, Xiaoling Dun ${ }^{1 *}$, Jinxing Tu${ }^{2}$, 5 and Hanzhong Wang ${ }^{1}$

6

$7{ }^{1}$ Oil Crops Research Institute of the Chinese Academy of Agricultural Sciences/Key Laboratory of 8 Biology and Genetic Improvement of Oil Crops, Ministry of Agriculture, Wuhan 430062, China

$9 \quad{ }^{2}$ National Key Laboratory of Crop Genetic Improvement, Huazhong Agricultural University, Wuhan 10 430062, China

11 These authors contributed equally to this work.

12 *Correspondence: dunxiaoling@,caas.cn

13 Email for Keqi Li: likeqi1218@sina.com; for Jie Wang: 123_wangjie@163.com; for Lieqiong Kuang: 14 396648536@qq.com; for Ze Tian: 917600094@qq.com; for Xinfa Wang: wangxinfa@caas.cn; $\underline{\text { for }}$ 15 Jinxing Tu: tujx@mail.hzau.edu.cn; for Hanzhong Wang: wanghz@oilcrops.cn 
Sixteen QTL clusters and four candidate genes controlling persistently root development, and thirty-two QTL clusters and eight candidate genes stage-specifically regulating root growth in rapeseed were detected in this study.

\section{Abstract}

Root growth is a dynamic process mediated by interactive genetic factors and environmental cues. Seven root-related traits and two shoot-related traits in 280 Brassica napus accessions at five continuous vegetative stages were measured to establish the genetic basis of root growth in rapeseed. The persistent and stage-specific genetic mechanisms were revealed by root dynamic analysis. Sixteen persistent and 32 stage-specific quantitative trait loci (QTL) clusters were identified through genome-wide association study (GWAS). Root samples with contrasting (slow and fast) growth rates throughout investigated stages and those with obviously stage-specific changes in growth rates were subjected to transcriptome analysis. The former identified 367 differentially expressed genes (DEGs) with persistently differential expressions throughout root development that were significantly enriched in GO terms of energy metabolism and response to biotic or abiotic stress, whereas the later identified 485 stage-specific DEGs with different expressions at corresponding stage that were enriched in GO terms of nitrogen metabolism. By integrating GWAS, weighted gene co-expression network analysis (WGCNA), and differential expression analysis, four and eight candidate genes less than $100 \mathrm{~kb}$ away from peak SNPs of QTL clusters were identified as key persistent and stage-specific genetic factors, respectively. The homologs of 3 genes (BnaA03g52990D, BnaA06g37280D, and BnaA09g07580D) out of 12 candidate genes have been reported to regulate root development in previous studies. Our results provide new insights into the temporal genetic mechanisms of root growth by identifying key candidate QTL/genes in rapeseed. 


\section{Introduction}

The root system architecture (RSA) usually denotes the spatial configuration of complex assembly of the root system, and root shape plays key role in plant healthy growth since root system penetrates the soil in search for water and nutrients (Giehl et al. 2014). Therefore, plants rely on the modulation of RSA in response to a changing soil environment. The genetic improvement of root architecture such as increasing lateral root (LR) number, facilitates resource bioavailability in plants and increases crop yield and stress tolerance (Uga et al. 2013; Chen et al. 2015).

To breed the crops with better RSA, a large number of studies have focused on variations in root architecture in many crops, such as rice, wheat, maize, soybean, and rapeseed (Burton et al. 2014; Song et al. 2016a; Beyer et al. 2019; Wang et al. 2019b; Guo et al. 2020). Several studies have identified hundreds of root QTL in controlled environments or in the field (Song et al. 2016b; Wang et al. 2017, 2019a, 2019b). Besides, several of these QTL have also been reported to influence such traits as yield, water/nutrient uptake, and abiotic stress tolerance (Hua et al. 2016; Song et al. 2016b; Gong and McDonald 2017; Fan et al. 2018; Bernardino et al. 2019; Schierholt et al. 2019; Islam et al. 2021).

Genome-wide association study (GWAS) has been successfully used for the identification of the polymorphism sites and/or genes related to complex traits including root traits in crops such as rice, wheat, maize, and rapeseed (Wang et al. 2017, 2019a; Sanchez et al. 2018; Beyer et al. 2019; Zhang et al. 2020). Moreover, brassinosteroid signaling kinase 3 (BSK3) was confirmed to regulate root elongation in low nitrogen in Arabidopsis by GWAS (Jia and Giehl 2019). Transcriptome analysis has become an effective technique for detecting candidate genes. Many crucial candidate DEGs related to root development have been identified by RNA sequencing in rice, maize, and B. napus (Dun et al. 2016; Hey et al. 2017; Xu and Zou 2019). Weighted gene co-expression network analysis (WGCNA) has been usually used to analyze the relationship and network between different genes. Functional candidate genes related to root development were identified at different developmental stages in crops by WGCNA, including DcMYB113, which was reported to regulate anthocyanin transport in carrot root (Xu et al. 2020), and three hub genes (GRMZM2G477658, GRMZM2G15536, and GRMZM2G072121) played a possible role in maize root formation and growth through the division and/or elongation of cells (Hwang et al. 2018). Recently, the combination of GWAS, transcriptome sequencing, and/or WGCNA has been turned out to be a rapid and efficient approach 
to identifying crucial candidate genes regulating root development (Li et al. 2017; Que et al. 2018; Wang et al. 2019a; Guo et al. 2020). For example, OsNall and OsJAZ1 located in the peak SNPs have been confirmed to facilitate the root development in rice (Li et al. 2017).

Rapeseed (Brassica napus L.; Brassicaceae), a globally cultivated crop, is one of the most important vegetable oil sources for human consumption. The Brassica 60K Illumina single-nucleotide polymorphism (SNP) array has facilitated the genetic improvement of different traits including flowering time, seed oil content, and phosphate-efficiency to obtain desirable alleles in B. napus (Liu et al. 2016, 2020; Wang et al. 2017). Root growth is a continuous and complex process with temporal dynamics and spatial patterning. A previous study has defined seven root growth types in a B. napus recombinant inbred line (RIL) population derived from two rapeseed cultivars (Zhongshuang11 and NO. 73290) with contrasting root systems, and identified two types of QTL (persistent and stage-specific) by the analysis of root traits in rapeseed (Wang et al. 2019b). To further identify the genetic factors controlling the dynamic root growth, we examined five continuous stages during root development in 280 natural accessions of B. napus. Sixteen persistent and 32 stage-specific QTL clusters further confirmed the existence of the two types of QTL controlling root development. In addition, we performed a transcriptome analysis of samples of four root growth types with extremely contrasting growth rates during the investigated time points. A total of 12 crucial candidate genes involved in root growth were identified via combining GWAS, WGCNA, and differential expression analysis, some of which have been reported to be related to root development in previous studies.

\section{Materials and methods}

\section{Plant materials and growth conditions}

The natural population used in this study consisted of 280 B. napus lines, including 156 semi-winter accessions, 86 spring accessions and 38 winter accessions. A total of the 280 rapeseed germplasm accessions were collected, including 222 accessions from the Yangtze River of China, 23 from northwestern China, 16 from Europe, 14 from Australia, and 5 from other places or unknown origins.

The previously reported hydroponic system was used for the root-related trait evaluation of the 280 B. napus accessions (Wang et al. 2019b). Briefly, uniform and stout rapeseed seeds were placed 
on the medical gauze of the germination device for $2 \mathrm{~d}$ in the dark, then they grew in the light (180 $\mu$ mol photons $\left.\mathrm{m}^{-2} \cdot \mathrm{s}^{-1}\right)$ for $4 \mathrm{~d}$ in a greenhouse $(60-80 \%$ relative humidity) under $16 / 8 \mathrm{~h}$ day/night cycles at $24^{\circ} \mathrm{C}$ (Fig. S1 A, B). A quarter of modified Hoagland's nutrient solution was filled into germination device to retain moisture and provide nutrients for seed germination (Hoagland et al., 1938). Six days after sowing, uniform seedlings were transferred to the growth device containing $1 / 4$ Hoagland's solution. The $1 / 4$ solution was replaced with a $1 / 2$ solution, and then with a $100 \%$ solution once a week until harvesting.

\section{Phenotypic evaluation of association panel}

The accessions from the natural population were completely randomly grown and evaluated with three independent biological replications. In each replication, samples (three uniform plants per accession) were collected from the germination device at the 13 days after sowing (DAS). At 6 DAS, 24 plants of an accession were transplanted to one growth device (Fig. S1C). Then three plants per accession were sampled from the growth device at four time points, namely, 10 days after transplanting (10 DAT, equal to $16 \mathrm{DAS}$ ), three expanding leaves (3 EL), $5 \mathrm{EL}$, and $7 \mathrm{EL}$, respectively. Once the plants were sampled, shoot fresh weight (SFW), root fresh weight (RFW), and primary root length (PRL) were measured manually. The intact roots were scanned and analyzed using WinRHIZO-Pro software (Regent Instruments, QC, Canada) to determine total root length (TRL), total root surface (TSA), total root volume (TRV), and total number of roots (TNR). Subsequently, shoot and root samples were dehydrated at $65^{\circ} \mathrm{C}$ for a week to determine shoot dry weight (SDW) and root dry weight (RDW). The above abbreviations used in this study were listed in Table 1.

\section{Data analysis}

The variance and correlation analyses of the investigated traits were performed using the software SAS 9.2. The broad-sense heritability was calculated using the formula reported by Liu et al. (2016). The principal component analysis (PCA) of all the investigated traits were conducted by the software SAS 9.2. The first step of PCA was to obtain the correlation matrix between different traits, then the dimensionality reduction was performed to obtain eight principal components, and PC1 and PC2 were plotted by R. According to previous reported method (Wang et al. 2019b), 
the growth rate (GR) of accession sample was calculated as the root fresh weight (RFW) value

130 at the late stage minus that at the early stage, and then divided by the growing days. GR of an 131 accession was normalized according to the following formula. Normalized GR $=(\mathrm{GRg}-$ $132 \mathrm{GRp} / \mathrm{GRp}$ In the formula, GRg represented the GR of a genotype, and GRp was the average GR of 133 the population of 280 accessions which were clustered in terms of the normalized GR by using 134 MeV_4_9_0 software (http://mev.ro/en/).

\section{Population structure, relative kinship, and association analysis}

The Brassica 60K Illumina ${ }^{\circledR}$ Infinium consortium SNP array (Clarke et al. 2016)

(http://www.illumina.com/technology/beadarray-technology/infinium-hd-assay.html) was used for accessions genotype. SNP data were analyzed using Illumina BeadStudio genotyping software (http://www.illumina.com/) with parameters set as a missing rate $\leqslant 0.2$, heterozygous rate $\leqslant 0.2$, and minor allele frequency $(\mathrm{MAF})>0.05$. BLAST was performed to search the probe sequences of these SNPs against the B.napus Darmor-bzh reference genome (Chalhoub et al. 2014) with an threshold of $\mathrm{e}^{-10}$. SNPs with merely one matched position in reference genome were used for further analysis. The population structure and relative kinship of the 280 B. napus accessions were analyzed using STRUCTURE v. 2.3.4 and SPAGeDi software, respectively (Pritchard et al. 2000). The linkage disequilibrium (LD) decay between all SNPs was assessed by TASSEL 4.0 (Bradbury et al. 2007) The trait-SNP association was analyzed by using the mixed linear model (MLM) (Yu and Buckler 2006). Marker haplotypes at each associated locus were identified using the four-gamete rule with Haploview software (Wei et al. 2016).

\section{Transcriptome sequencing and analysis}

Five accessions from each of the four growth types (type 1, type 2, type 4, and type 5, detailed information presented in result section) with contrasting GRs were selected and replanted for further transcriptome analysis. Total roots of three plants for each accession were sampled at four time points

153 (10 DAT, 3 EL, 5 EL, and 7 EL) with two biological replications. Samples of the five accessions within 154 one growth type at each sampling time point with the same weight were mixed as a single sample. A 155 total of 32 obtained samples were fully mixed for total RNA extraction with the IRIzol reagent 156 (Invitrogen, USA). Sequencing library construction and Illumina sequencing were performed by the 
Oebiotech Company in Shanghai, China using an Illumina HiSeq ${ }^{\mathrm{TM}} 2500$ platform. The raw reads with 150 paired-end base pair (bp) were filtered and aligned as previously reported (Dun et al., 2016). The raw data were submitted into database of the National Center for Biotechnology Information Sequence Read Archive (SRA; http://www.ncbi.nlm.nih.gov/sra) (Accession No. PRJNA714285).

The clean reads were mapped to the B.napus Darmor-bzh reference genome (Chalhoub et al. 2014) (http://www.genoscope.cns.fr/brassicanapus/data/) using Hisat2. The gene expression levels were expressed as FPKM (fragments per kilobase per million reads) value. The PCA of the gene expressions was performed using the PCAtools package in R. The WGCNA was conducted using the WGCNA package in $\mathrm{R}$ (Langfelder and Horvath 2008). $\mathrm{P} \leqslant 0.05$ for the false discovery rate (FDR) and $\left|\log _{2}{ }^{\text {ratio }}\right|$ $\geqslant 1$ were used as criteria to identify DEGs with the DESeq package in R. The k-mean clustering was performed by MeV_4_9_0 software. Gene ontology (GO) enrichment analysis was performed using the ClusterProfiler package in $\mathrm{R}$.

\section{Real-time reverse transcription PCR}

Quantitative real-time PCR (qRT-PCR) of 20 genes randomly selected from the DEGs was performed to verify the accuracy of RNA-seq data. The primer sequences were presented in Table S1. The SYBR qPCR Master Mix (Vazyme) was used for qRT-PCR analysis with the CFX96 (BIO-RAD). Three technical replications were performed for each sample. The B. napus ACTIN2 was used as a internal control to compute the relative expression of target genes by the $2^{-\Delta \Delta \mathrm{CT}}$ method.

\section{Results}

\section{Phenotypic analysis of $280 \mathrm{~B}$. napus accessions reveals genetic stability of root development}

To examine dynamic growth patterns of roots during the vegetative stage, the hydroponic system was used for evaluating root-related traits and shoot biomass traits of $280 \mathrm{~B}$. napus accessions which were sampled at 13 DAS from the germination device and at 10 DAT, 3 EL, 5 EL, and 7 EL from the growth device with three biological replications for each sample, respectively (Fig. S1 D-H). The statistics of the seven root-related traits (RFW, PRL, TRL, TSA, TRV, TNR, and RDW), and two shoot biomass traits (SFW and SDW) from each replication at the five sampling time points were listed in Table S2, and the mean values of three replications were presented in Table 2. All the investigated traits showed a normal distribution or approximate normal distribution (Fig. S2). Coefficient of variation (CV) values ranging from $15.21 \%$ to $25.66 \%$, indicating considerable phenotypic variations for all the traits in the 
population (Table 2). All the traits showed a high broad-sense heritability $\left(\mathrm{H}^{2}\right)$, ranging from 0.68 to 0.94. For root traits, RFW, TRL, TSA, and TRV had heritability slightly higher than TNR (Table 2). We discovered significant correlations of each trait among all five stages with $\mathrm{r}^{2}$ ranging from 0.33 to 0.87 $(\mathrm{P}<0.0001)$. In general, the highest root correlations were observed between two adjacent stages in spite of the gradually decreased correlation with the increased sampling interval, indicating that the effects of environment on these traits increased with plant development (Fig. 1). The PCA results of the traits suggested that component $1(47.9 \%)$ and component $2(13.0 \%)$ explained the majority of genetic trait variation in this population (Fig. 2). With exception of PRL (Group 1), all the traits examined at early stages (13 DAS and 10 DAT) were clustered into Group 3, whereas the traits recorded at late stages (3 EL, 5 EL, and 7 EL) were clustered into another group (Group 2). Traits captured at early stages and late stages were separated by component 2 , but mapped to the same position on component 1, suggesting the high correlations between early stages and late stages (Fig. 2). As shown in Table S3, all the traits were significantly correlated with $\mathrm{r}^{2}$ ranging from 0.24 to $0.74(\mathrm{P}<0.001)$ among the three biological replications. The results suggested that early root development traits were positively correlated with late root traits, thus suggesting that root development was a continuous process influenced by early genetic factors.

\section{Persistent and stage-specific QTL clusters related to the root system are identified by GWAS}

After filtering, a total of 23,542 SNPs with known physical position in the B.napus Darmor-bzh reference genome were selected for GWAS (Chalhoub et al. 2014). The distribution of the 23,542 SNP markers and LD decay on each chromosome were presented in Table S4. Approximately 58.1\% of the kinship coefficients between individual accessions were equal to zero, and $97.6 \%$ were less than 0.2 , suggesting a weak kinship for most accessions in the natural population (Fig. S3).

The 1,107 significant trait-SNP associations were detected $\left(-\log _{10} \mathrm{P}>4.37,-\log _{10}{ }^{1 / 23,542}\right)$ by using the MLM (Table S5). We termed the SNPs with close proximity (within $1 \mathrm{Mb}$ ) and an LD $\mathrm{r}^{2}>0.2$ as one cluster since these SNPs were identified as the same QTL (Liu et al. 2016). As a result, a total of 683 identified significant trait-SNP associations with 134 significant SNPs markers, and 747 suggestive trait-SNP associations $\left(3.5<-\log _{10} \mathrm{P} \leqslant 4.37\right)$ were integrated into 48 valid QTL clusters (Table S6, Fig. 3). Of these 48 clusters, 21 QTL clusters contained multiple SNPs and 27 QTL clusters harboured single SNPs. The maximum genetic variation explained by these clusters ranged 
2165 EL, and 7 EL stages, respectively. Except 8 QTL clusters S1, S2, \#1, S6, S8, S21, S26, and S18, all

217 other clusters (40 out of 48) were detected at two or more stages. Noteworthy, two significant SNPs

218 displaying the vast majority of trait-SNP associations on chromsome C8 were detected at 13 DAS,

21910 DAT, 5 EL and 7 EL (Fig. 4). This suggested the existence of genetic factors controlling multiple

220 root-related traits at various stages.

221 To reveal the genetic basis of root traits at the multiple vegetative stages, these QTL clusters were divided into three categories based on their identification stages: ES-specific clusters (early stage, 13 DAS and 10 DAT), LS-specific clusters (later stage, 3 EL, 5 EL, and 7 EL), and ES-LS common clusters. Sixteen out of the 48 QTL clusters constituted ES-LS common clusters, indicating the existence of the persistent QTL controlling root development. Additionally, 16 ES-specific clusters and 16 LS-specific clusters revealed genetic mechanism in the root system at specific stages (Table S6, Fig. 2). The major QTL identified in this study could be applied for improving root system architecture in rapeseed.

\section{Transcriptome analysis reveals dynamic root development}

230

Clustering analysis of the 280 accessions was performed to examine the similarity and diversity of their root growth patterns. At the same the growth stage, the traits (except PRL) exhibited significant correlations ( $\mathrm{P}<0.0001$ ) with each other (from 0.44 to $0.97, \mathrm{P}<0.0001$; Table $\mathrm{S} 7$ ), suggesting developmental relevance among these root-related traits. SFW was considered as the trait reflecting the plant growth status. RFW showed higher correlations with SFW $(0.70-0.79)$ than with other root traits (Table S7). The traits investigated at 13 DAS and 10 DAT were from different growth device (germination device and growth device), so GRs from 13 DAS to 10 DAT weren't shown in this study. The normalized GRs were calculated by RFW to present the root dynamic growth patterns, the heatmap showed that the 280 accessions fell into seven growth types (Type 1-7) (Fig. 5A). The 38 accessions (accounting for 13.57\%) belonged to growth type 1 with their GRs below the average GR from 10 DAT to $7 \mathrm{EL}$, and at least one GR less than $80 \%$ of the average. The 48 accessions (17.14\%) belonged to growth type 2 with their GRs greater than the average GR from 10 DAT to 7 EL, and at least one GR greater than $120 \%$ of the average. The majority of the accessions (64, $22.86 \%$ ) belonged to type 3 with a relatively stable GR ranging from $80 \%$ to $120 \%$ of the average 
GR from 10 DAT to 7 EL. Type 4 possessed 48 accessions (17.14\%) whose GRs were below average

GR from 10 DAT to $3 \mathrm{EL}$ or from $10 \mathrm{DAT}$ to $5 \mathrm{EL}$, but were above average from $3 \mathrm{EL}$ to $7 \mathrm{EL}$ or from 5 EL to 7 EL. Contrastive, type 5 consisted of 50 accessions (17.86\%) whose GRs were above average GR from 10 DAT to $3 \mathrm{EL}$ or from $10 \mathrm{DAT}$ to $5 \mathrm{EL}$, then below average from $3 \mathrm{EL}$ to $7 \mathrm{EL}$ or from 5 EL to 7 EL (Fig. 5A and B). Type 6 contained 17 accessions (6.07\%) with its GR changing from fast to slow, and then to fast again during the investigated stages. On the contrary, the GRs of type 7 consisting of 15 accessions (5.36\%) were subjected to the change pattern of first slow, and then fast, followed by slow. Obviously, the majority of accessions fell into type 1 (with consistent slow GR) and type 2 (with consistent fast GR), and type 3 (with stable GR), indicating that genes expressed at an early stage might control root growth at the late stage with prolonged effects. Additionally, type 4 and type 5 displayed obvious stage-specific changes in GRs, suggesting the existence of genes functioning at a specific stage.

The GRs of type 3 were close to average GR. The GRs of type 6 and type 7 exhibit two reverse changes during root development. Considering this, we excluded type 3, 6, and 7 in subsequent transcriptome analysis. We selected the four growth types (type 1, type 2, type 4, and type 5) for subsequent transcriptome analysis because type 1 and type 2 had contrasting (slow and fast) GRs throughout root development stage, type 4 and type 5 exhibited opposite changing GRs at the specific stage. Five accessions from each growth type were sampled at the 10 DAT, 3 EL, 5 EL, and 7 EL stage, respectively (Fig. 5C), and were subjected to transcriptome analysis to reveal the temporal molecular mechanisms of root development. A total of more than 41 million clean reads were obtained from each library after adaptor trimming, of which, $73.47 \%-91.59 \%$ clean reads were uniquely matched to B. napus reference genome (Table S8). The qRT-PCR of 20 genes was performed in all the samples (Table S1). The results of qRT-PCR were highly consistent with those of RNA-Seq data, suggesting the reliability of the RNA-Seq data (Fig. S4). The PCA of the RNA-Seq data indicated that all the four root growth types displayed an obvious separation between group 1 (10 DAT and 3 EL stage) and group 2 (5 EL and 7 EL stage) on component 1 (Fig. S5), indicating a change in gene expression from 3 EL to 5 EL during root development. 
To explore the persistent genetic factors during root development, the VENN analysis of the DEGs from growth type 1 Vs type 2 at the four root development stages was performed. A total of 367 DEGs were found to be overlapped within the four stages (Table S9). A K-means clustering analysis of these persistent DEGs showed that the expressions of these genes were stabilized among the four MYB_related, MADS-box, and E2F/DP. The genes BnaAnng09810D, BnaA01g20660D, BnaC03g61210D homologous to the MADS-box family member ARABIDOPSIS NITRATE REGULATED 1, ANR1, the NAC transcription factor family Peroxidase 34, PER34, and bHLH25, respectively, which have been reported to participate in root development (Gaudinier et al. 2018; Sun et al. 2018; Chutia et al. 2019), were expressed higher at all the stags of type 1 than type 2 . The GO enrichment analysis showed that the 367 DEGs were enriched in GO terms related to energy metabolism (including acetyl-CoA biosynthetic process from pyruvate and glycolytic process) and biotic or abiotic stress (such as response to oxidative stress, hydrogen peroxide catabolic process and cold acclimation) (Fig. 6C, Table S10).

Meanwhile, 485 stage-specific DEGs were identified, which exhibited lower or higher expressions at type 4 early stages than at late stages, and displayed opposite expression patterns at type 5 corresponding stages (Fig. 6B, Table S11). The GO enrichment analysis revealed that these genes were significantly enriched in GO terms of nitrate metabolism (including nitrate transport, response to nitrate, nitrate assimilation, and cellular response to nitrogen starvation) and plant-type cell wall organization (Fig. 6D, Table S12). In this study, a total of 16 stage-specific DEGs were highly and Wada 2018; Zhang et al. 2019). These results suggested that the biological processes, energy metabolism, and response to biotic or abiotic stress might influence the persistent root development, 
development was also regulated by several important transcription factors. expression analysis

303 To investigate the gene regulatory network during root development, 26,039 DEGs from the four root growth types were used to identify co-expression gene modules by WGCNA. A total of 30 modules were identified in the dendrogram according to correlations of genes (Fig. 7A), and the relationships between modules and samples were presented in Fig. 7B. The purple module was associated with all the stages of type 1 , whereas the green module was associated with all the stages of type 2. The darkorange, darkturquoise, white, and darkred modules were significantly associated with 10 DAT, 3 EL, 5 EL and 7 EL of growth type 4, respectively, and the red, lightyellow, saddlebrown and darkgrey modules exhibited high correlations with 10 DAT, 3 EL, 5 EL and 7 EL of type 5, respectively. The heatmaps showed that the genes within one module were highly expressed in the samples highly correlated with the module (Fig. S6). GWAS results indicated that 2,461 genes were located in the haplotype blocks of the 48 QTL clusters (Table S13). Considering a high correlation of WGCNA genes with each module $\left(\mathrm{r}^{2}>0.85\right), 9$ persistent and 13 stage-specific candidate genes each including 3 DEGs were screened from GWAS and WGCNA overlapped genes (Table 3 and Table 4). In addition, 6 GWAS and DEGs overlapped genes with correlations to the modules $<0.85$ were also screened as stage-specific candidate genes (Table 4). purple and green modules were highly expressed at all the stages of type 1 and type 2, respectively (Table 3). Four genes in the green module were located less than $100 \mathrm{~kb}$ away from the peak SNPs, including BnaA05g03210D, BnaC02g10480D, BnaA03g52990D, and BnaC08g39040D which were 35.9 Kb, 50.6 Kb, 69.3 Kb, and 6.3 Kb apart from the peak SNPs of \#4, \#9, S20, and S26 (Table 3). BnaA03g52990D encodes the GATA transcription factor, whose homolog influences root development by affecting auxin level and cell division in Arabidopsis (Jiang et al. 2018). Two genes BnaC02g10710D and BnaA05g03210D exhibited high correlation with BnaA03g52990D (Fig. 8A). ROOT INITIATION DEFECTIVE 1, RID1 (the homolog of BnaC02g10710D) has been reported to function in root apical meristem and root morphogenesis in Arabidopsis (Ohtani et al. 2013). Furthermore, three persistent DEGs, BnaC02g10480D, BnaC02g10710D, and BnaA05g03210D, 
displayed high correlations to each other in the green module (Fig. 8A).

330 Of the 19 stage-specific candidate genes, BnaA03g47900D in the white module and seven genes 331 BnaA03g42930D, BnaA09g07840D, BnaC01g22700D, BnaC02g14450D, BnaC03g26110D, 332 BnaA06g37280D, and BnaC02g14330D in the red module were located less than $100 \mathrm{~kb}$ away from 333 the peak SNPs (Table 4). Especially, BnaC01g22700D encoding ferredoxin-3 protein was $0.06 \mathrm{~kb}$ 334 apart from the peak SNP of S19 QTL cluster. Our data indicated that PROTEIN BASIC 335 PENTACYSTEINE5, BPC5 (the homologs of BnaA06g37280D) was located $28.6 \mathrm{~kb}$ away from the 336 peak SNP of \#11, and in previous study, BPC5 has been found to promote lateral root (LR) growth in 337 Arabidopsis (Mu et al. 2017). Our WGCNA, stage-specific DEG analysis, and GWAS results 338 indicated that BnaA03g42930D, BnaA09g07840D, and BnaC01g22700D were detected and were 339 highly expressed at 10 DAT of type 5 (Table 4), and that these three genes were highly correlated 340 with another three closely-linked genes BnaA08g24190D, BnaC02g14450D, and BnaC03g26110D 341 (Fig. 8B).

342 In the present study, four and eight genes with less than $100 \mathrm{~kb}$ physical distances from the peak SNPs were screened as crucial persistent and stage-specific candidate genes in B.napus. Furthermore, homologs of three candidate genes (BnaA03g52990D, BnaA06g37280D, and BnaA09g07580D) have been reported to regulate root development in previous studies. The results showed that the method of screening candidate genes by combining GWAS, WGCNA, and differential expression analysis was effective.

\section{Discussion}

Two types (persistent and stage-specific) of temporal genetic factors controlling root development in B. napus

351 Recent advances in high-resolution imaging of root growth have indicated that the root system was determined by continuous spatial and temporal growth (Thomas et al. 2016a, b; Pulwicki and Hobill 2017; Wang et al. 2019b). Consistent with the previous report (Wang et al. 2019b), our phenotypic correlation analyses and root growth dynamics study revealed two types (persistent and stage-specific) of temporal genetic factors controlling root development in B. napus. Further, the persistent and stage-specific genetic factors were verified by our identified QTL clusters and DEGs 
on several dynamic traits at different developmental stages in Arabidopsis, barley, wheat, upland cotton, maize, and B. napus (Liang et al. 2014; Bac-Molenaar et al. 2015; Wang et al. 2015; Muraya et al. 2017; Su et al. 2018; Du et al. 2019; Mohler and Stadlmeier 2019; Knoch et al. 2020). For example, 35 dynamic conditional QTL which can enhance the number of roots were detected at different root development stages in upland cotton, suggesting the dynamic development of roots (Liang et al. 2014).

Furthermore, the peak SNPs of 18 QTL clusters in this study were co-localized in the identical haplotype blocks of the 27 previously reported significant SNPs related to root traits at low or sufficient phosphorus conditions (Table S14) (Wang et al. 2017). Clusters S10, S20, \#15, and S25 were also co-localized with previously identified QTL (qcA09-1, qcC02-2, qcC02-2, and uqPRLC06) related to root surface area (RSA) trait, respectively (Wang et al. 2017, 2019b). Our results provided useful QTL and the major QTL can be used for marker-assisted selection of root traits in rapeseed.

\section{Possible regulatory pathways of persistent and stage-specific genetic factors related to root} development

Root growth, as a complex process, is determined by the interaction of many genes. Some genes play a persistent role during root development, whereas others function at a specific stage. In this study, we identified 367 persistent DEGs from growth type 1 vs type 2 controlling root development in rapeseed. Three persistent DEGs enriched in acetyl-CoA biosynthetic process were homologs of Pyruvate dehydrogenase E1, ALPHA in A. thaliana affecting polar auxin transport during root development (Ohbayashi and Huang 2019). Oxidative stress response is a general response of living organisms to biotic or abiotic stress (Piacentini et al. 2019). Ten out of the 14 persistent DEGs (Table S9) enriched in oxidative stress encoded 10 proteins PEROXIDASE 34 (PRX34), CYP709B3, CYP87A3, CYP78A6, PEROXIDASE 3 (PER3), PER34, PER44, PER71, CATALASE-2 (CAT2), and ALPHA-DIOXYGENASE 1 (DOX1), and these genes have been reported to act as regulators in roots development in Arabidopsis (Takei et al. 2004; Kim et al. 2010; Renault et al. 2013; Estornell et al. 2018; Renard et al. 2020). For example, $P R X 34$ mediated $\mathrm{H}_{2} \mathrm{O}_{2}$ generation and increased $\mathrm{Ca}^{2+}$ flux from the cytosol of Atmpk6 root cells to inhibit root elongation (Han et al. 2015). CYP709B subfamily was involved in cytokinin metabolism and signaling in roots (Takei et al. 2004). These results suggested that biological processes, such as energy metabolism and biotic or abiotic stress 
response, especially oxidative stress response might act as the major molecule mechanisms influencing persistent root development.

$\mathrm{NO}^{3-}$ and nitrate metabolites can serve as regulatory signals to control root system architecture (Bouguyon et al. 2015). Three stage-specific DEGs (Table S11) homologous to NRT1/NPF6.3 not only regulated auxin biosynthesis to promote LR primordia emergence, but also repressed LR development by promoting auxin transport at low nitrate in Arabidopsis (Maghiaoui et al. 2020). High-affinity nitrate transporter 2.1 (NRT2.1) homologous to our four stage-specific DEGs has been reported to play an essential role in root nitrate uptake (Table S11) (Jacquot et al. 2020). The 485 stage-specific DEGs were found to be enriched in GO terms of nitrate transport, response to nitrate, and nitrate assimilation (Fig. 6, Table S12). The above results suggested that nitrate metabolism process might mainly regulate the stage-specific root development.

Efficient discovery of candidate genes by combining GWAS, WGCNA, and differential expression analysis

Combination of GWAS, WGCNA, and differential expression analysis has been reported as an efficient way to acquire crucial genes in maize, rice, soybean, carrot, and other crops (Li et al. 2017; Que et al. 2018; Wang et al. 2019a; Guo et al. 2020). We identified four persistent and eight stage-specific crucial candidate genes related to root development by integrating GWAS, WGCNA and differential expression analysis in rapeseed.

Four crucial persistent candidate genes BnaA03g52990D, BnaA05g03210D, BnaC02g10480D, BnaC08g39040D in the green module displayed high correlations to each other (Fig. 8), and two genes, BnaA03g52990D and BnaC02g10710D in green module were homologous to ATGATA3 and ATRID1 which have been reported to function in root development in Arabidopsis (Ohtani et al. 2013; Jiang et al. 2018). Our identified ATRID1 had similar function with SRD2 which affected LR morphogenesis by reducing the level of auxin efflux facilitator (PIN) in A. thaliana (Ohtani et al. 2010, 2013). Furthermore, the homologs of other genes in the green module, Protein phosphatase 2C (AIP1), Replication protein A subunit B (RPA1B), Histidine kinase 3 (AHK3), Polyadenylate-binding protein 2 (PAB2), have also been reported to affect root development by regulating phytohormone or promoting cell elongation (Riefler et al. 2006; Lan et al. 2011; Lim et al. 2012; Markakis et al. 2012). All these results indicated that the crucial persistent candidate genes in 
the green module might have similar functions during root development.

417 Seven out of eight stage-specific crucial candidate genes were in the red module, of which 418 BnaA06g37280D and BnaA09g07580D were homologous to BPC5 and RALFL34 reported to 419 promote LR development by inhibiting the abscisic acid insensitive 4 expression and activating PIN1 level in Arabidopsis (Murphy et al. 2016; Mu et al. 2017). Moreover, the red module included several function-known genes involved in root development, such as BnaA08g06170D and BnaC08g06550D which were homologous to AtSMAP1 reported to modulate root development by interacting with 2,4-Dichlorophenoxyacetic acid (Takahashi et al. 2017). The results above further demonstrated that these seven candidate genes played significant roles in root growth. to broaden our research on molecular mechanism of root development. More studies are needed to further analyze these candidate genes and validate their functions.

Acknowledgements This work was supported by the National Key Research and Development and Technology Innovation Project (CAAS-ASTIP-2013-OCRI), and Rapeseed Industry Technology System (CARS-13).

Author contribution statement All the authors planned and supervised the research. KQL, JW, LQK, $\mathrm{ZT}, \mathrm{XFW}$, and JXT performed root traits investigation and analyzed the data; KQL wrote the manuscript; XLD, and HZW contributed to modify the manuscript.

\section{Compliance with ethical standards}

Conflict of interest Authors state that there is no conflict of interest. 


\section{References}

Bac-Molenaar JA, Vreugdenhil D, Granier C, Keurentjes JJ (2015) Genome-wide association mapping of growth dynamics detects time-specific and general quantitative trait loci. J Exp Bot 66:5567-5580. doi:10.1093/jxb/erv176

Bernardino KC et al. (2019) The genetic architecture of phosphorus efficiency in sorghum involves pleiotropic QTL for root morphology and grain yield under low phosphorus availability in the soil. BMC Plant Biol 19:87. doi:10.1186/s12870-019-1689-y

Beyer S, Daba S, Tyagi P, Bockelman H, Brown-Guedira G, Mohammadi M (2019) Loci and candidate genes controlling root traits in wheat seedlings-a wheat root GWAS. Funct Integr Genomics 19:91-107. doi:10.1007/s10142-018-0630-z

Bouguyon E et al. (2015) Multiple mechanisms of nitrate sensing by Arabidopsis nitrate transceptor NRT1.1. Nat Plants 1:15015. doi:10.1038/nplants.2015.15

Bradbury PJ, Zhang Z, Kroon DE, Casstevens TM, Yogesh R, Buckler ES (2007) TASSEL: software for association mapping of complex traits in diverse samples. Bioinformatics:19:2633-2635. doi:10.1093/bioinformatics/btm308

Burton AL et al. (2014) QTL mapping and phenotypic variation for root architectural traits in maize (Zea mays L.). Theor Appl Genet 127:2293-2311. doi:10.1007/s00122-014-2353-4

Chalhoub B et al. (2014) Plant genetics. Early allopolyploid evolution in the post-Neolithic Brassica napus oilseed genome. Science 345:950-953. doi:10.1126/science.1253435

Chen YS, Lo SF, Sun PK, Lu CA, Ho TH, Yu SM (2015) A late embryogenesis abundant protein HVA1 regulated by an inducible promoter enhances root growth and abiotic stress tolerance in rice without yield penalty. Plant Biotechnol J 13:105-116. doi:10.1111/pbi.12241

Chutia R, Abel S, Ziegler J (2019) Iron and Phosphate Deficiency Regulators Concertedly Control Coumarin Profiles in Arabidopsis thaliana Roots During Iron, Phosphate, and Combined Deficiencies. Front Plant Sci 10:113. doi:10.3389/fpls.2019.00113

Clarke WE et al. (2016) A high-density SNP genotyping array for Brassica napus and its ancestral diploid species based on optimised selection of single-locus markers in the allotetraploid genome. Theor Appl Genet 129:1887-1899. doi:10.1007/s00122-016-2746-7

Du B et al. (2019) Mapping dynamic QTL dissects the genetic architecture of grain size and grain filling rate at different grain-filling stages in barley. Sci Rep 9:18823. doi:10.1038/s41598-019-53620-5

Dun X, Tao Z, Wang J, Wang X, Liu G, Wang H (2016) Comparative transcriptome analysis of primary roots of Brassica napus seedlings with extremely different primary root lengths using RNA sequencing. Front Plant Sci 7:1238. doi:10.3389/fpls.2016.01238

Estornell LH, Landberg K, Cierlik I, Sundberg E (2018) SHI/STY genes affect pre- and post-meiotic anther processes in auxin sensing domains in Arabidopsis. Front Plant Sci 9:150. doi:10.3389/fpls.2018.00150

Fan X et al. (2018) Identification of QTL regions for seedling root traits and their effect on nitrogen use efficiency in wheat (Triticum aestivum L.). Theor Appl Genet 131:2677-2698. doi:10.1007/s00122-018-3183-6

Gaudinier A et al. (2018) Transcriptional regulation of nitrogen-associated metabolism and growth. Nature 563:259-264. doi:10.1038/s41586-018-0656-3

Giehl RF, Gruber BD, von Wiren N (2014) It's time to make changes: modulation of root system architecture by nutrient signals. J Exp Bot 65:769-778. doi:10.1093/jxb/ert421 
Gong X, McDonald G (2017) QTL mapping of root traits in phosphorus-deficient soils reveals important genomic regions for improving NDVI and grain yield in barley. Theor Appl Genet 130:1885-1902. doi:10.1007/s00122-017-2931-3

Guo J et al. (2020) Transcriptome and GWAS analyses reveal candidate gene for seminal root length of maize seedlings under drought stress. Plant Sci 292:110380. doi:10.1016/j.plantsci.2019.110380

Han S, Fang L, Ren X, Wang W, Jiang J (2015) MPK6 controls $\mathrm{H}^{2} \mathrm{O}^{2}$-induced root elongation by mediating $\mathrm{Ca}^{2+}$ influx across the plasma membrane of root cells in Arabidopsis seedlings. New Phytol 205:695-706. doi:10.1111/nph.12990

Hey S et al. (2017) Complexity and specificity of the maize (Zea mays L.) root hair transcriptome. J Exp Bot 68:2175-2185. doi:10.1093/jxb/erx104

Hua Y, Zhang D, Zhou T, He M, Ding G, Shi L, Xu F (2016) Transcriptomics-assisted quantitative trait locus fine mapping for the rapid identification of a nodulin 26-like intrinsic protein gene regulating boron efficiency in allotetraploid rapeseed. Plant, Cell \& Environ 39:1601-1618. doi:10.1111/pce.12731

Hwang SG, Kim KH, Lee BM, Moon JC (2018) Transcriptome analysis for identifying possible gene regulations during maize root emergence and formation at the initial growth stage. Genes Genomics 40:755-766. doi:10.1007/s13258-018-0687-z

Islam A et al. (2021) Fine mapping and candidate gene analysis of qRN5a, a novel QTL promoting root number in rice under low potassium. Theor Appl Genet 134:213-227. doi:10.1007/s00122-020-03692-z

Jacquot A et al. (2020) NRT2.1 C-terminus phosphorylation prevents root high affinity nitrate uptake activity in Arabidopsis thaliana. New Phytol 228:1038-54. doi:10.1111/nph.16710

Jia Z, Giehl RFH (2019) Natural variation of BSK3 tunes brassinosteroid signaling to regulate root foraging under low nitrogen. Nature Commun 10:2378. doi:10.1038/s41467-019-10331-9

Jiang K, Yung V, Chiba T, Feldman LJ (2018) Longitudinal patterning in roots: a GATA2-auxin interaction underlies and maintains the root transition domain. Planta 247:831-843. doi:10.1007/s00425-017-2831-4

Kim MJ, Ciani S, Schachtman DP (2010) A peroxidase contributes to ROS production during Arabidopsis root response to potassium deficiency. Mol Plant 3:420-427. doi: $10.1093 / \mathrm{mp} / \mathrm{ssp} 121$

Knoch D et al. (2020) Strong temporal dynamics of QTL action on plant growth progression revealed through high-throughput phenotyping in canola. Plant Biotechnol J 18:68-82. doi:10.1111/pbi.13171

Lan P, Li W, Wen TN, Shiau JY, Wu YC, Lin W, Schmidt W (2011) iTRAQ protein profile analysis of Arabidopsis roots reveals new aspects critical for iron homeostasis. Plant Physiol 155:821-834. doi:10.1104/pp.110.169508

Langfelder P, Horvath S (2008) WGCNA: an R package for weighted correlation network analysis. BMC Bioinform 9:559. doi:10.1186/1471-2105-9-559

Li X et al. (2017) Genetic control of the root system in rice under normal and drought stress conditions by genome-wide association study. PLoS Genet 13:e1006889. doi:10.1371/journal.pgen.1006889

Liang Q et al. (2014) Dynamic QTL and epistasis analysis on seedling root traits in upland cotton. J Genet 93:63-78. doi:10.1007/s12041-014-0341-8 
Lim CW, Kim JH, Baek W, Kim BS, Lee SC (2012) Functional roles of the protein phosphatase 2C, AtAIP1, in abscisic acid signaling and sugar tolerance in Arabidopsis. Plant Sci 187:83-88. doi:10.1016/j.plantsci.2012.01.013

Liu S, Fan C, Li J, Cai G, Zhou Y (2016) A genome-wide association study reveals novel elite allelic variations in seed oil content of Brassica napus. Theor Appl Genet 1:1203-1215. doi:10.1007/s00122-016-2697-z

Liu S et al. (2020) Dissection of genetic architecture for glucosinolate accumulations in leaves and seeds of Brassica napus by genome-wide association study. Plant Biotechnol J 18:1472-1484. doi:10.1111/pbi.13314

Maghiaoui A et al. (2020) The Arabidopsis NRT1.1 transceptor coordinately controls auxin biosynthesis and transport to regulate root branching in response to nitrate. $\mathrm{J}$ Exp Bot 71:4480-94. doi:10.1093/jxb/eraa242

Markakis MN et al. (2012) Identification of genes involved in the ACC-mediated control of root cell elongation in Arabidopsis thaliana. BMC Plant Biol 12:208. doi:10.1186/1471-2229-12-208

Mohler V, Stadlmeier M (2019) Dynamic QTL for adult plant resistance to powdery mildew in common wheat (Triticum aestivum L.). J Appl Genet 60:291-300. doi:10.1007/s13353-019-00518-7

$\mathrm{Mu} \mathrm{Y}$ et al. (2017) BASIC PENTACYSTEINE proteins repress ABSCISIC ACID INSENSITIVE4 expression via direct recruitment of the polycomb-repressive complex 2 in Arabidopsis root development. Plant, Cell \& Environ 58:607-621. doi:10.1093/pcp/pcx006

Muraya MM, Chu J, Zhao Y, Junker A, Klukas C, Reif JC, Altmann T (2017) Genetic variation of growth dynamics in maize (Zea mays L.) revealed through automated non-invasive phenotyping. Plant J 89:366-380. doi:10.1111/tpj.13390

Murphy E et al. (2016) RALFL34 regulates formative cell divisions in Arabidopsis pericycle during lateral root initiation. J Exp Bot 67:4863-4875. doi:10.1093/jxb/erw281

Ohbayashi I, Huang S (2019) Mitochondrial pyruvate dehydrogenase contributes to auxin-regulated organ development. Plant Physiol 180:896-909. doi:10.1104/pp.18.01460

Ohtani M, Demura T, Sugiyama M (2010) Particular significance of SRD2-dependent snRNA accumulation in polarized pattern generation during lateral root development of Arabidopsis. Plant \& Cell Physiol 51:2002-2012. doi:10.1093/pcp/pcq159

Ohtani M, Demura T, Sugiyama M (2013) Arabidopsis root initiation defective1, a DEAH-box RNA helicase involved in pre-mRNA splicing, is essential for plant development. Plant Cell 25:2056-2069. doi:10.1105/tpc.113.111922

Piacentini D, Falasca G, Canepari S, Massimi L (2019) Potential of PM-selected components to induce oxidative stress and root system alteration in a plant model organism. Environ. Int 132:105094. doi:10.1016/j.envint.2019.105094

Pritchard JK, Stephens M, Donnelly P (2000) Inference of population structure using multilocus genotype data. Genetics 155:945-959.

Pulwicki J, Hobill D (2017) The dynamics of root growth: a geometric model. Bull of Mathe Biol 79:1820-1845. doi:10.1007/s11538-017-0308-Z

Que F, Wang GL, Li T, Wang YH, Xu ZS, Xiong AS (2018) Genome-wide identification, expansion, and evolution analysis of homeobox genes and their expression profiles during root development in carrot. Funct Integr Genomics 18:685-700. doi:10.1007/s10142-018-0624-x

Renard J et al. (2020) $P R X 2$ and $P R X 25$, peroxidases regulated by $C O G 1$, are involved in seed 
longevity in Arabidopsis. Plant, Cell \& Environ 43:315-326. doi:10.1111/pce.13656

Renault H, El Amrani A, Berger A, Mouille G, Soubigou-Taconnat L, Bouchereau A, Deleu C (2013) $\gamma$-Aminobutyric acid transaminase deficiency impairs central carbon metabolism and leads to cell wall defects during salt stress in Arabidopsis roots. Plant, Cell \& Environ 36:1009-1018. doi:10.1111/pce.12033

Riefler M, Novak O, Strnad M, Schmülling T (2006) Arabidopsis cytokinin receptor mutants reveal functions in shoot growth, leaf senescence, seed size, germination, root development, and cytokinin metabolism. Plant Cell 18:40-54. doi:10.1105/tpc.105.037796

Sanchez DL, Liu S, Ibrahim R, Blanco M, Lubberstedt T (2018) Genome-wide association studies of doubled haploid exotic introgression lines for root system architecture traits in maize (Zea mays L.). Plant Sci 268:30-38. doi:10.1016/j.plantsci.2017.12.004

Schierholt A, Tietz T, Bienert GP, Gertz A, Miersch S, Becker HC (2019) Root system size response of bzh semi-dwarf oilseed rape hybrids to different nitrogen levels in the field. Ann Bot 124:891-901. doi:10.1093/aob/mcy197

Song L et al. (2016a) Genome-wide transcriptome analysis of soybean primary root under varying water-deficit conditions. BMC Genomics 17:57. doi:10.1186/s12864-016-2378-y

Song W, Wang B, Hauck AL, Dong X, Li J, Lai J (2016b) Genetic dissection of maize seedling root system architecture traits using an ultra-high density bin-map and a recombinant inbred line population. J Integr Plant Biol 58:266-279. doi:10.1111/jipb.12452

Su Y, Li M, Guo L (2018) Different effects of phospholipase D $\zeta 2$ and non-specific phospholipase C4 on lipid remodeling and root hair growth in Arabidopsis response to phosphate deficiency. Plant J 94:315-326. doi:10.1111/tpj.13858

Sun CH et al. (2018) Chrysanthemum MADS-box transcription factor CmANR1 modulates lateral root development via homo-/heterodimerization to influence auxin accumulation in Arabidopsis. Plant Sci 266:27-36. doi:10.1016/j.plantsci.2017.09.017

Takahashi M, Umetsu K, Oono Y, Higaki T, Blancaflor EB, Rahman A (2017) Small acidic protein 1 and SCF(TIR)(1) ubiquitin proteasome pathway act in concert to induce 2,4-dichlorophenoxyacetic acid-mediated alteration of actin in Arabidopsis roots. Plant $\mathrm{J}$ 89:940-956. doi:10.1111/tpj.13433

Takei K, Yamaya T, Sakakibara H (2004) Arabidopsis CYP735A1 and CYP735A2 encode cytokinin hydroxylases that catalyze the biosynthesis of trans-Zeatin. J Biol Chem 279:41866-41872. doi:10.1074/jbc.M406337200

Thomas CL et al. (2016a) Root morphology and seed and leaf ionomic traits in a Brassica napus L. diversity panel show wide phenotypic variation and are characteristic of crop habit. BMC Plant Biol 16:214. doi:10.1186/s12870-016-0902-5

Thomas CL et al. (2016b) High-throughput phenotyping (HTP) identifies seedling root traits linked to variation in seed yield and nutrient capture in field-grown oilseed rape (Brassica napus L.). Ann Bot 118:655-665. doi:10.1093/aob/mcw046

Tominaga-Wada R, Wada T (2018) CPC-ETC1 chimeric protein localization data in Arabidopsis root epidermis. Data in Brief 18:1773-1776. doi:10.1016/j.dib.2018.04.055

Uga Y et al. (2013) Control of root system architecture by DEEPER ROOTING 1 increases rice yield under drought conditions. Nat Genet 45:1097-1102. doi:10.1038/ng.2725

Wang $\mathrm{H}$ et al. (2019a) Integrating GWAS and gene expression analysis identifies candidate genes for root morphology traits in maize at the seedling stage. Genes (Basel) 10:773. 
Wang J, Kuang L, Wang X, Liu G, Dun X, Wang H (2019b) Temporal genetic patterns of root growth in Brassica napus L. revealed by a low-cost, high-efficiency hydroponic system. Theor Appl Genet 132:2309-2323. doi:10.1007/s00122-019-03356-7

Wang X et al. (2017) Genetic variants associated with the root system architecture of oilseed rape (Brassica napus L.) under contrasting phosphate supply. DNA Res 24:407-417. doi:10.1093/dnares/dsx013

Wang X et al. (2015) Dynamic and comparative QTL analysis for plant height in different developmental stages of Brassica napus L. Theor Appl Genet 128:1175-1192. doi:10.1007/s00122-015-2498-9

Wei L et al. (2016) Genome-wide association analysis and differential expression analysis of resistance to Sclerotinia stem rot in Brassica napus. Plant Biotechnol J 14:1368-1380. doi:10.1111/pbi.12501

Xu Y, Zou J (2019) RNA-Seq transcriptome analysis of rice primary roots reveals the role of flavonoids in regulating the rice primary root growth. Genes (Basel) 10:213. doi:10.3390/genes10030213

Xu ZS, Yang QQ, Feng K, Yu X, Xiong AS (2020) DcMYB113, a root-specific R2R3-MYB, conditions anthocyanin biosynthesis and modification in carrot. Plant Biotech J 18:1585-1597. doi:10.1111/pbi.13325

Yu J, Buckler ES (2006) Genetic association mapping and genome organization of maize. Current Opinion in Biotechnology 17:155-160. doi:10.1016/j.copbio.2006.02.003

Zhang $\mathrm{H}$ et al. (2020) Genome-wide association study of root system development at seedling stage in rice. Genes (Basel) 11:1395. doi:10.3390/genes11121395

Zhang J et al. (2019) Transcriptional regulatory framework for vascular cambium development in 639 
641 Table 1 Abbreviations of investigated traits used in this study

\begin{tabular}{|c|c|c|}
\hline Abbreviation & The full name & Description \\
\hline 13 DAS & 13 days after sowing & Sampled at 13 days after sowing from the germination device \\
\hline $10 \mathrm{DAT}$ & ten days after transferring & Sampled at ten days after transplanting from the growth device \\
\hline $3 \mathrm{EL}$ & three expending leaves & Sampled at three expending leaves from the growth device \\
\hline $5 \mathrm{EL}$ & five expending leaves & Sampled at five expending leaves from the growth device \\
\hline $7 \mathrm{EL}$ & seven expending leaves & Sampled at seven expending leaves from the growth device \\
\hline PRL & Primary root length & Primary root length \\
\hline RDW & Root dry weight & Root dry weight \\
\hline RFW & Root fresh weight & Root fresh weight \\
\hline SDW & Shoot dry weight & Shoot dry weight \\
\hline SFW & Shoot fresh weight & Shoot fresh weight \\
\hline TNR & Total root number & Total root number \\
\hline TRL & Total root length & Total root length \\
\hline TRV & Total root volume & Total root volume \\
\hline TSA & Total root surface area & Total root surface area \\
\hline
\end{tabular}

642 
Table 2 Nine trait statistics of 280 accessions collected at five continuous stages

\begin{tabular}{|c|c|c|c|c|c|c|c|c|c|c|}
\hline Traits & Environment & Min & Max & Mean & SD & $\mathrm{CV}(\%)$ & $\sigma \mathrm{g}^{2}$ & $\sigma \mathrm{g} \times \mathrm{e}^{2}$ & $\sigma^{2}$ & $\mathrm{H}^{2}$ \\
\hline \multirow{5}{*}{$\begin{array}{l}\text { PRL, } \\
\mathrm{cm}\end{array}$} & 13DAS & 4.85 & 18.48 & 11.22 & 2.14 & 19.06 & 52.74 & 10.95 & 2.45 & 0.93 \\
\hline & 10DAT & 8.36 & 27.81 & 17.08 & 3.12 & 18.28 & 74.37 & 19.65 & 6.31 & 0.91 \\
\hline & $3 \mathrm{EL}$ & 11.23 & 28.16 & 17.87 & 2.96 & 16.58 & 78.47 & 23.98 & 8.87 & 0.90 \\
\hline & $5 \mathrm{EL}$ & 11.87 & 27.71 & 18.91 & 2.88 & 15.21 & 74.52 & 19.45 & 8.00 & 0.91 \\
\hline & 7EL & 13.03 & 31.30 & 20.09 & 3.10 & 15.44 & 85.20 & 20.93 & 7.79 & 0.92 \\
\hline \multirow{5}{*}{$\begin{array}{c}\text { SFW, } \\
\mathrm{g}\end{array}$} & 13DAS & 0.21 & 0.94 & 0.52 & 0.11 & 20.95 & 0.129 & 0.020 & 0.009 & 0.94 \\
\hline & 10DAT & 0.42 & 2.06 & 1.24 & 0.27 & 21.53 & 0.450 & 0.112 & 0.020 & 0.92 \\
\hline & $3 \mathrm{EL}$ & 1.22 & 4.58 & 2.95 & 0.56 & 18.97 & 2.794 & 0.816 & 0.207 & 0.90 \\
\hline & $5 \mathrm{EL}$ & 3.87 & 17.21 & 10.92 & 1.91 & 17.51 & 32.64 & 8.86 & 2.53 & 0.85 \\
\hline & 7EL & 10.20 & 39.97 & 25.12 & 4.40 & 17.53 & 171.56 & 71.56 & 12.56 & 0.87 \\
\hline \multirow{5}{*}{$\begin{array}{c}\text { RFW, } \\
\mathrm{g}\end{array}$} & 13DAS & 0.031 & 0.152 & 0.084 & 0.020 & 23.23 & 0.003 & 0.001 & 0.000 & 0.93 \\
\hline & 10DAT & 0.046 & 0.270 & 0.175 & 0.039 & 22.03 & 0.010 & 0.003 & 0.001 & 0.90 \\
\hline & $3 \mathrm{EL}$ & 0.218 & 0.839 & 0.487 & 0.097 & 19.84 & 0.083 & 0.026 & 0.008 & 0.90 \\
\hline & $5 \mathrm{EL}$ & 0.629 & 2.754 & 1.621 & 0.335 & 20.64 & 1.004 & 0.371 & 0.087 & 0.81 \\
\hline & 7EL & 1.24 & 5.42 & 3.07 & 0.63 & 20.45 & 3.52 & 1.45 & 0.286 & 0.87 \\
\hline \multirow{5}{*}{$\begin{array}{c}\text { SDW, } \\
\text { mg }\end{array}$} & 13DAS & 9.89 & 46.42 & 27.03 & 5.88 & 21.75 & 291.57 & 73.0 & 40.6 & 0.74 \\
\hline & 10DAT & 23.89 & 128.9 & 74.57 & 15.79 & 21.18 & 2488.4 & 752.8 & 390.3 & 0.84 \\
\hline & $3 \mathrm{EL}$ & 61.78 & 250.3 & 160.9 & 32.63 & 20.28 & 9387.8 & 3590.5 & 1069.1 & 0.88 \\
\hline & $5 \mathrm{EL}$ & 231.1 & 1067 & 659.7 & 117.4 & 17.79 & 123001 & 53857 & 12285 & 0.80 \\
\hline & 7EL & 647.8 & 2482.2 & 1632.9 & 306.4 & 18.77 & 825608 & 434176 & 77621 & 0.84 \\
\hline \multirow{5}{*}{$\begin{array}{c}\text { RDW, } \\
\mathrm{mg}\end{array}$} & 13DAS & 1.58 & 6.11 & 3.69 & 0.83 & 22.43 & 2.03 & - & 0.50 & - \\
\hline & 10DAT & 2.44 & 13.09 & 8.45 & 1.68 & 19.92 & 71.87 & 55.53 & 84.98 & 0.68 \\
\hline & $3 \mathrm{EL}$ & 10.00 & 30.78 & 19.71 & 3.83 & 19.44 & 115.5 & 49.3 & 19.2 & 0.81 \\
\hline & $5 \mathrm{EL}$ & 29.11 & 98.78 & 61.26 & 11.42 & 18.65 & 1169 & 479.3 & 127.7 & 0.76 \\
\hline & 7EL & 59.00 & 294.3 & 143.4 & 33.06 & 23.06 & 8580 & 4926 & 804.3 & 0.82 \\
\hline \multirow{5}{*}{$\begin{array}{l}\text { TRL, } \\
\mathrm{cm}\end{array}$} & 13DAS & 50.03 & 294.0 & 160.5 & 36.86 & 22.96 & 11290 & 2049 & 934.16 & 0.93 \\
\hline & 10DAT & 142.2 & 772.2 & 457.3 & 104.5 & 22.86 & 31861 & 11146 & 2112 & 0.89 \\
\hline & $3 \mathrm{EL}$ & 495.7 & 1337 & 778.1 & 139.6 & 17.94 & 172904 & 43177 & 15901 & 0.91 \\
\hline & $5 \mathrm{EL}$ & 980.3 & 2679 & 1671 & 297 & 17.77 & 784719 & 260737 & 68229 & 0.89 \\
\hline & 7EL & 1262.9 & 5134 & 2967 & 601 & 20.26 & 3192567 & 1348006 & 280825 & 0.87 \\
\hline \multirow{5}{*}{$\begin{array}{l}\text { TSA, } \\
\mathrm{cm}^{2}\end{array}$} & 13DAS & 4.68 & 19.65 & 11.23 & 2.40 & 21.38 & 45.61 & 8.39 & 4.36 & 0.93 \\
\hline & 10DAT & 8.11 & 43.33 & 27.91 & 5.69 & 20.39 & 116.64 & 41.60 & 9.13 & 0.89 \\
\hline & $3 \mathrm{EL}$ & 34.81 & 93.31 & 59.95 & 9.56 & 15.94 & 812.0 & 221.1 & 89.99 & 0.91 \\
\hline & $5 \mathrm{EL}$ & 85.83 & 224.0 & 146.2 & 24.0 & 16.40 & 5090 & 1603 & 485.5 & 0.90 \\
\hline & 7EL & 116.2 & 483.7 & 268.1 & 51.9 & 19.35 & 23836 & 10067 & 2025 & 0.87 \\
\hline \multirow{5}{*}{$\begin{array}{l}\text { TRV, } \\
\mathrm{cm}^{3}\end{array}$} & 13DAS & 0.025 & 0.119 & 0.063 & 0.014 & 22.20 & 0.002 & 0.000 & 0.000 & 0.93 \\
\hline & 10DAT & 0.037 & 0.211 & 0.137 & 0.028 & 20.13 & 0.003 & 0.001 & 0.000 & 0.89 \\
\hline & $3 E L$ & 0.185 & 0.570 & 0.374 & 0.063 & 16.98 & 812.0 & 221.1 & 89.99 & 0.91 \\
\hline & $5 \mathrm{EL}$ & 0.482 & 1.774 & 1.030 & 0.189 & 18.33 & 0.315 & 0.100 & 0.029 & 0.90 \\
\hline & 7EL & 0.859 & 3.639 & 1.947 & 0.402 & 20.63 & 1.427 & 0.584 & 0.112 & 0.87 \\
\hline \multirow{5}{*}{ TNR } & 13DAS & 60.52 & 233.3 & 121.3 & 26.3 & 21.71 & 11974 & 4531 & 972 & 0.88 \\
\hline & 10DAT & 139.2 & 673.5 & 382.8 & 82.3 & 21.49 & 26044 & 10602 & 1977 & 0.87 \\
\hline & $3 \mathrm{EL}$ & 284.7 & 924.8 & 521.5 & 93.7 & 17.96 & 77915 & 22475 & 8438 & 0.90 \\
\hline & $5 \mathrm{EL}$ & 603.3 & 2169 & 1273 & 265.2 & 20.84 & 623914 & 319736 & 61184 & 0.85 \\
\hline & 7EL & 1175 & 5318 & 2759 & 708.0 & 25.66 & 4394488 & 2543179 & 405276 & 0.83 \\
\hline
\end{tabular}

644 Min, minimum of values in the population; Max, maximum of values; Mean, mean trait value; SD, Standard Deviation of trait

645 values; $\mathrm{CV}$, coefficient of variation. $\sigma \mathrm{g}^{2}, \sigma \mathrm{g} \times \mathrm{e}^{2}$ and $\sigma^{2}$ estimated variance associated with the effect of genotype, genotype $\times$

646 environment and the residual error, respectively $(P<0.0001)$. $\mathrm{H}^{2}$, broad-sense heritability. 
Table 3 Key persistent candidate genes identified by GWAS, WGCNA, and differential expression analysis

\begin{tabular}{|c|c|c|c|c|c|c|c|c|c|c|c|c|}
\hline Gene ID & Cluster & $\begin{array}{l}\text { Distance to } \\
\text { peak SNP }(\mathrm{Kb})\end{array}$ & $\begin{array}{l}\text { Type1_1 } \\
\text { DAT }\end{array}$ & $\begin{array}{l}\text { Type1_3 } \\
\text { EL }\end{array}$ & $\begin{array}{l}\text { Type1_5 } \\
\text { EL }\end{array}$ & $\begin{array}{l}\text { Type1_7 } \\
\text { EL }\end{array}$ & $\begin{array}{l}\text { Type2_1 } \\
\text { ODAT }\end{array}$ & $\begin{array}{l}\text { Type2_3 } \\
\text { EL }\end{array}$ & $\begin{array}{l}\text { Type2_5 } \\
\text { EL }\end{array}$ & $\begin{array}{l}\text { Type2_7 } \\
\text { EL }\end{array}$ & gene_symbol & Description \\
\hline \multicolumn{13}{|l|}{ purple } \\
\hline BnaA05g11320D & S7 & 342.28 & 4.43 & 1.89 & 3.13 & 3.72 & 1.89 & 1.64 & 1.64 & 1.48 & PCMP-E75 & Pentatricopeptide repeat-containing protein \\
\hline $\begin{array}{l}\text { BnaA10g24040D } \\
\text { green }\end{array}$ & S17 & 236.56 & 4.53 & 5.54 & 5.48 & 4.13 & 3.50 & 3.14 & 3.54 & 1.15 & - & - \\
\hline BnaA05g03210D & $\# 9$ & 35.9 & 9.80 & 4.22 & 6.64 & 5.89 & 26.85 & 52.49 & 36.34 & 36.96 & EPSIN2 & Clathrin interactor EPSIN 2 \\
\hline BnaC02g10480D $\mathrm{D}^{\mathrm{a}}$ & S20 & 50.6 & 1.80 & 0.78 & 0.15 & 0.56 & 16.07 & 42.07 & 16.13 & 31.31 & SBT4.8 & Subtilisin-like serine endopeptidase family protein \\
\hline $\mathrm{BnaC} 02 \mathrm{~g} 10710 \mathrm{D}^{\mathrm{a}}$ & S20 & 157.3 & 0.07 & 0.23 & 0.27 & 0.27 & 4.07 & 5.84 & 3.18 & 3.94 & RID1 & ROOT INITIATION DEFECTIVE 1 \\
\hline BnaA02g20510D & S1 & 170.99 & 8.96 & 5.62 & 5.06 & 5.02 & 17.57 & 10.61 & 11.67 & 15.96 & RSH1 & Putative GTP diphosphokinase RSH1 \\
\hline BnaA03g52990D ${ }^{b}$ & \#4 & 69.3 & 3.36 & 7.90 & 0.89 & 2.10 & 11.78 & 14.47 & 9.05 & 12.04 & GATA3 & GATA transcription factor 3 (GATA3) \\
\hline BnaC08g35330D $\mathrm{D}^{\mathrm{b}}$ & $\# 21$ & 168.29 & 206.76 & 116.64 & 58.63 & 134.22 & 332.26 & 443.97 & 227.90 & 382.19 & - & - \\
\hline BnaC08g39040D ${ }^{b}$ & S26 & 6.28 & 7.63 & 5.94 & 5.02 & 8.72 & 18.90 & 22.82 & 17.99 & 14.99 & PSS1 & CDP-diacylglycerol--serine O-phosphatidyltransferase 1 \\
\hline
\end{tabular}

648 a: candidate genes overlapped by WGCNA, GWAS and DEGs, b: candidate genes overlapped by WGCNA and GWAS 


\begin{tabular}{|c|c|c|c|c|c|c|c|c|c|c|c|c|}
\hline Gene ID & Cluster & $\begin{array}{l}\text { Distance to peak } \\
\text { SNP }(\mathrm{Kb})\end{array}$ & $\begin{array}{l}\text { Type4 } \\
\text { DAT }\end{array}$ & $\begin{array}{l}\text { Type4_3 } \\
\text { EL }\end{array}$ & $\begin{array}{l}\text { Type4_5 } \\
\text { EL }\end{array}$ & $\begin{array}{l}\text { Type4_7 } \\
\text { EL }\end{array}$ & $\begin{array}{l}\text { Type5_1 } \\
\text { ODAT }\end{array}$ & $\begin{array}{l}\text { Type5_3 } \\
\text { EL }\end{array}$ & $\begin{array}{l}\text { Type5_5 } \\
\text { EL }\end{array}$ & $\begin{array}{l}\text { Type5_7 } \\
\text { EL }\end{array}$ & gene_symbol & Description \\
\hline \multicolumn{13}{|l|}{ darkorange } \\
\hline BnaA05g22690D & S9 & 409.40 & 12.06 & 2.92 & 3.27 & 1.33 & 1.61 & 0.88 & 7.62 & 0.16 & - & - \\
\hline \multicolumn{13}{|l|}{ white } \\
\hline BnaA03g43140D ${ }^{b}$ & $\# 3$ & 106.11 & 1.28 & 1.19 & 3.24 & 1.16 & 0.37 & 0.03 & 0.07 & 0.00 & FCAALL.41 & Putative glycerol-3-phosphate transporter 4 \\
\hline BnaA03g47900D ${ }^{b}$ & S3 & 34.26 & 0.00 & 0.35 & 2.79 & 0.85 & 0.66 & 0.41 & 0.00 & 0.27 & - & - \\
\hline BnaA09g07790D $\mathrm{D}^{\mathrm{b}}$ & S10 & 107.12 & 0.66 & 0.70 & 2.66 & 0.99 & 0.65 & 0.39 & 0.27 & 0.18 & DAR3 & Protein DA1-related 3 \\
\hline $\begin{array}{l}\mathrm{BnaC} 08 \mathrm{~g} 33940 \mathrm{D}^{\mathrm{b}} \\
\text { red }\end{array}$ & \multicolumn{11}{|c|}{ red } & Cytoplasmic 60 S subunit biogenesis factor REI1 homolog 2 \\
\hline BnaA03g42930D $\mathrm{D}^{\mathrm{a}}$ & $\# 3$ & 32.11 & 2.07 & 4.87 & 1.50 & 2.05 & 13.84 & 1.41 & 0.31 & 1.28 & - & - \\
\hline BnaA09g07840D & S10 & 89.43 & 2.24 & 3.90 & 1.15 & 3.02 & 12.80 & 5.08 & 1.66 & 1.95 & PSBO1 & Oxygen-evolving enhancer protein 1-1 \\
\hline $\mathrm{BnaC} 01 \mathrm{~g} 22700 \mathrm{D}^{\mathrm{a}}$ & S19 & 0.06 & 3.35 & 4.12 & 4.70 & 5.38 & 22.74 & 7.92 & 4.62 & 4.68 & FD3 & Ferredoxin-3 \\
\hline BnaA03g54270D ${ }^{b}$ & $\# 5$ & 252.24 & 0.39 & 0.44 & 0.46 & 0.41 & 4.20 & 1.38 & 0.63 & 0.75 & - & - \\
\hline BnaA05g05400D ${ }^{b}$ & S6 & 114.77 & 22.97 & 21.13 & 26.99 & 30.04 & 58.49 & 33.78 & 26.45 & 37.25 & PRA1B2 & PRA1 family protein B2 \\
\hline BnaA08g24190D ${ }^{b}$ & $\# 13$ & 274.89 & 1.63 & 2.80 & 1.19 & 1.59 & 10.52 & 4.34 & 1.44 & 1.20 & - & - \\
\hline BnaC02g14450D ${ }^{b}$ & $\# 15$ & 92.11 & 2.23 & 0.00 & 0.86 & 1.19 & 85.14 & 0.04 & 0.55 & 1.62 & AGP22 & Arabinogalactan peptide 22 \\
\hline BnaC03g26110D & S22 & 59.91 & 20.84 & 8.96 & 13.42 & 18.16 & 44.32 & 19.22 & 17.27 & 12.82 & F4P13.7 & Universal stress protein A-like protein \\
\hline BnaA06g37280D & $\# 11$ & 28.60 & 5.36 & 5.99 & 5.64 & 4.03 & 16.82 & 5.83 & 4.99 & 4.92 & BPC5 & Protein basic pentacysteine 5 \\
\hline BnaA07g02950D & $\# 12$ & 253.94 & 1.35 & 0.72 & 2.20 & 4.45 & 6.65 & 1.41 & 2.66 & 4.18 & TIM14-3 & Mitochondrial import inner membrane translocase subunit \\
\hline BnaA09g07580D ${ }^{c}$ & S10 & 183.41 & 2.15 & 5.44 & 3.24 & 3.15 & 18.16 & 11.78 & 2.75 & 2.20 & RALFL34 & Protein RALF-like 34 \\
\hline $\mathrm{BnaC} 02 \mathrm{~g} 14330 \mathrm{D}^{\mathrm{c}}$ & $\# 15$ & 26.80 & 2.86 & 31.35 & 35.15 & 7.11 & 102.64 & 35.21 & 21.90 & 7.83 & - & - \\
\hline \multicolumn{13}{|l|}{ lightyellow } \\
\hline BnaA10g23820D $D^{b}$ & S17 & 133.94 & 3.46 & 7.57 & 6.16 & 7.00 & 7.91 & 27.73 & 15.74 & 10.48 & IPK2a & Inositol polyphosphate multikinase alpha \\
\hline
\end{tabular}

651 a: candidate gene overlapped by WGCNA, GWAS and DEGs, b: candidate gene overlapped by WGCNA and GWAS, c: candidate gene overlapped by GWAS and DEGs. 
Fig.1 Correlations of each captured trait at five stages. / represents the correlation, for example, 13DAS/10DAT represent the correlation in the traits between 13 DAS and 10 DAT, $P<0.0001$.

Fig. 2 Principal component analysis (PCA) of captured traits at five stages in association population. Three obvious groups are indicated with blue circles. PRL trait was clustered into group 1. Other traits captured at $13 \mathrm{DAS}$ and $10 \mathrm{DAT}$ were clustered into group 3. The traits captured at 3 EL, 5 EL, and 7 EL were clustered into group 2.

Fig. 3 Genetic landscapes of QTL clusters obtained in this study. QTL clusters refer to significant SNPs with close proximity (within $1 \mathrm{Mb}$ ) and an $\mathrm{LD}$ of $\mathrm{r}^{2}>0.2$. Position, physical range, number, largest genetic variation explanation of significant SNPs of each cluster. \# represents clusters with multiple SNPs. S indicates the clusters harbouring single SNPs. ES-specific clusters: early stage specific clusters, including 13 DAS and 10 DAT; LS-specific clusters: late stage specific clusters, including $3 \mathrm{EL}, 5 \mathrm{EL}$, and $7 \mathrm{EL}$; ES-LS common clusters: early and late stage clusters, including 13 DAS, 10 DAT, 3 EL, 5 EL, and 7 EL.

Fig. 4 Manhattan plot of total surface area (TSA) at five stages. The different colours represent the TSA trait-related SNPs at 13 DAS, 10 DAT, 3 EL, 5 EL, and 7 EL, respectively. The horizontal black lines indicate the significance threshold of GWAS $\left(-\log _{10} 1 / 23,542=4.37\right)$. The $\mathrm{x}$ axis shows the 19 chromosomes (A1-A10 and C1-C9) in B.napus. Each chromosome is scaled by the physical chromosome length.

Fig. 5 Clustering analyses of 280 accessions. (A) Hierarchical heatmap of 7 dynamic growth types of 280 accessions based on normalized GR values calculated by RFW. Red represents the GRs above the average, and green indicates the GRs below the average. (B) Diagram of 7 growth types with six accessions in each growth type. (C) Diagram of 4 transcriptome-sequenced growth types (type 1, 2, 4, and 5) with 5 accessions in each type.

Fig. 6 Information of persistent and stage-specific DEGs during root development in rapeseed.

677 (A) K-mean clustering analysis of persistent DEGs in type 1 and type 2. The $\mathrm{x}$ axis indicates the samples at the four stages of type 1 and type 2 . The $y$ axis denotes the $\log _{2}{ }^{(\text {FPKM) }}$. error bars represent the min and max data. (B) K-mean clustering analysis of the stage-specific expression genes in type 
denotes the $\log _{2}(\mathrm{FPKM})$. error bars represent the min and max data. (C) GO terms enriched with the persistent DEGs in type 1 and type 2. (D) GO terms enriched with the stage-specific expression genes in type 4 and type 5.

Fig. 7 WGCNA of gene expression matrix in rapeseed. (A) Hierarchical clustering tree (dendrogram) of genes based on co-expression network analysis. (B) Module-sample association. Each row corresponds to a module labeled with a color identical to that in (A), and each column corresponds to a sample.

Fig. 8 Networks of genes in green and red modules. (A-B) Correlation networks in green and red modules, respectively. Yellow colour in the network indicates the candidate genes overlapped by GWAS, WGCNA and differential expression analysis, and the purple colour in the network indicates the candidate genes overlapped by GWAS and WGCNA.

Supplemental Fig. S1 Phenotype of plants at different stages in B. napus. (A) Materials sowed on germination device. (B) Plants in germination device six day after sowing. (C) Plants in growth device. (D-H) Plants at 13 DAS, 10 DAT, 3 EL, 5 EL, and 7 EL, respectively.

Supplemental Fig. S2 Frequency distribution of root-related traits and shoot-related traits at five stages. (A-I) Frequency distribution of SFW, RFW, SDW, RDW, TNR, PRL, TRL, TSA, and TRV at the five stages (13 DAS, 10 DAT, 3 EL, 5 EL, and 7 EL), respectively.

Supplemental Fig. S3 Analysis of population structure and kinships of 280 B. napus accessions (A) Log likelihood data (LnP[D]) of possible clusters, K: from 1 to 10. (B) Distribution of pairwise relative kinship. (C) Population structure of 280 accessions.

Supplemental Fig. S4 Positive correlation between RNA-seq data and qRT-PCR data Supplemental Fig. S5 Principal component analysis of the transcriptome sequencing data Supplemental Fig. S6 Heatmap of module eigengenes obtained by WGCNA. (A-L) Heatmaps of the expression profile of eigengenes in the purple, green, black, brown, darkorange, darkturquoise, white, darkred, red, lightyellow, saddlebrown and darkgrey modules, respectively. 
Supplemental Fig. S1 Phenotype of plants at different stages in B. napus

Supplemental Fig. S2 Frequency distribution of root-related traits and shoot-related traits at five

711 stages

712 Supplemental Fig. S3 Analyses of population structure and kinships of 280 B. napus accessions

713 Supplemental Fig. S4 Positive correlation between RNA-seq data and qRT-PCR data

714 Supplemental Fig. S5 Principal component analysis of the transcriptome sequencing data.

715 Supplemental Fig. S6 Heatmap of module eigengenes obtained by WGCNA.

716 Supplemental Table S1 Primers used in this study

717 Supplemental Table S2 Trait statistics collected at the five stages of each repetition

718 Supplemental Table S3 Correlations in each captured trait among replication at five stages

719 Supplemental Table S4 Summary of SNPs and LD decay on 19 chromosomes of B. napus

720 Supplemental Table S5 Detailed information on trait-related significant SNPs identified by GWAS

721 Supplemental Table S6 Detailed information on 48 valid QTL clusters

722 Supplemental Table S7 Correlations among root-related traits at each examined stage

723 Supplemental Table S8 RNA-Seq statistics of four growth types against B. napus reference 724 genome.

725 Supplemental Table S9 FPKM of persistent DEGs

726 Supplemental Table S10 GO enrichment results of persistent DEGs

727 Supplemental Table S11 FPKM of stage-specific DEGs

728 Supplemental Table S12 GO enrichment results of stage-specific expressed genes

729 Supplemental Table S13 FPKM of genes located in haplotype blocks on 48 QTL clusters

730 Supplemental Table S14 Information on peak SNPs overlapped with SNPs reported by Wang et al. $731 \quad(2017)$ 


\section{Figures}

$\begin{array}{lllllllllll}\text { TNR } & 0.7 & 0.4 & 0.36 & 0.33 & 0.55 & 0.47 & 0.42 & 0.58 & 0.4 & 0.65\end{array}$

$\begin{array}{lllllllllll}\text { TRV } & 0.82 & 0.58 & 0.46 & 0.46 & 0.61 & 0.51 & 0.47 & 0.71 & 0.6 & 0.76\end{array}$

$\begin{array}{lllllllllll}\text { TSA } & 0.82 & 0.56 & 0.48 & 0.47 & 0.54 & 0.45 & 0.44 & 0.71 & 0.58 & 0.77\end{array}$

$\begin{array}{llllllllllll}\text { TRL } & 0.79 & 0.55 & 0.5 & 0.47 & 0.49 & 0.43 & 0.45 & 0.73 & 0.56 & 0.76 & \text { Corr }\end{array}$

$\begin{array}{llllllllllll}\text { RDW } & 0.75 & 0.4 & 0.44 & 0.4 & 0.53 & 0.56 & 0.43 & 0.71 & 0.49 & 0.7 & 0.5 \\ \end{array}$

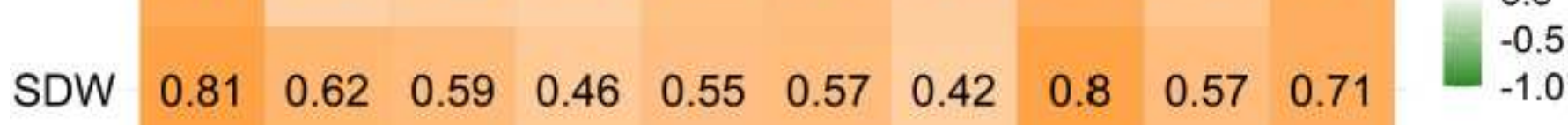

$\begin{array}{lllllllllll}\text { RFW } & 0.79 & 0.55 & 0.49 & 0.48 & 0.49 & 0.43 & 0.45 & 0.74 & 0.61 & 0.75\end{array}$

$\begin{array}{lllllllllll}\text { SFW } & 0.85 & 0.67 & 0.57 & 0.5 & 0.63 & 0.54 & 0.47 & 0.82 & 0.66 & 0.79\end{array}$

$\begin{array}{lllllllllll}\text { PRL } & 0.78 & 0.75 & 0.72 & 0.69 & 0.69 & 0.66 & 0.6 & 0.87 & 0.81 & 0.87\end{array}$

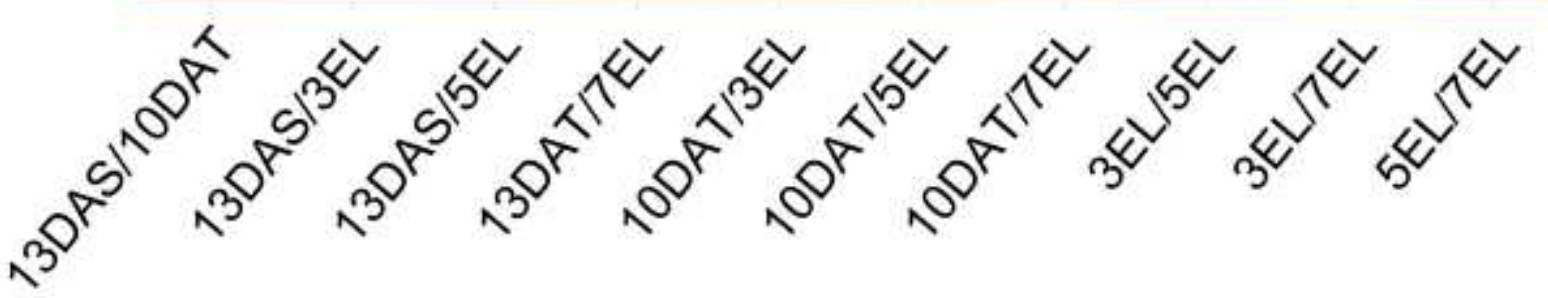

\section{Figure 1}

Correlations of each captured trait at five stages. / represents the correlation, for example, 13DAS/10DAT represent the correlation in the traits between 13 DAS and 10 DAT, $P<0.0001$. 


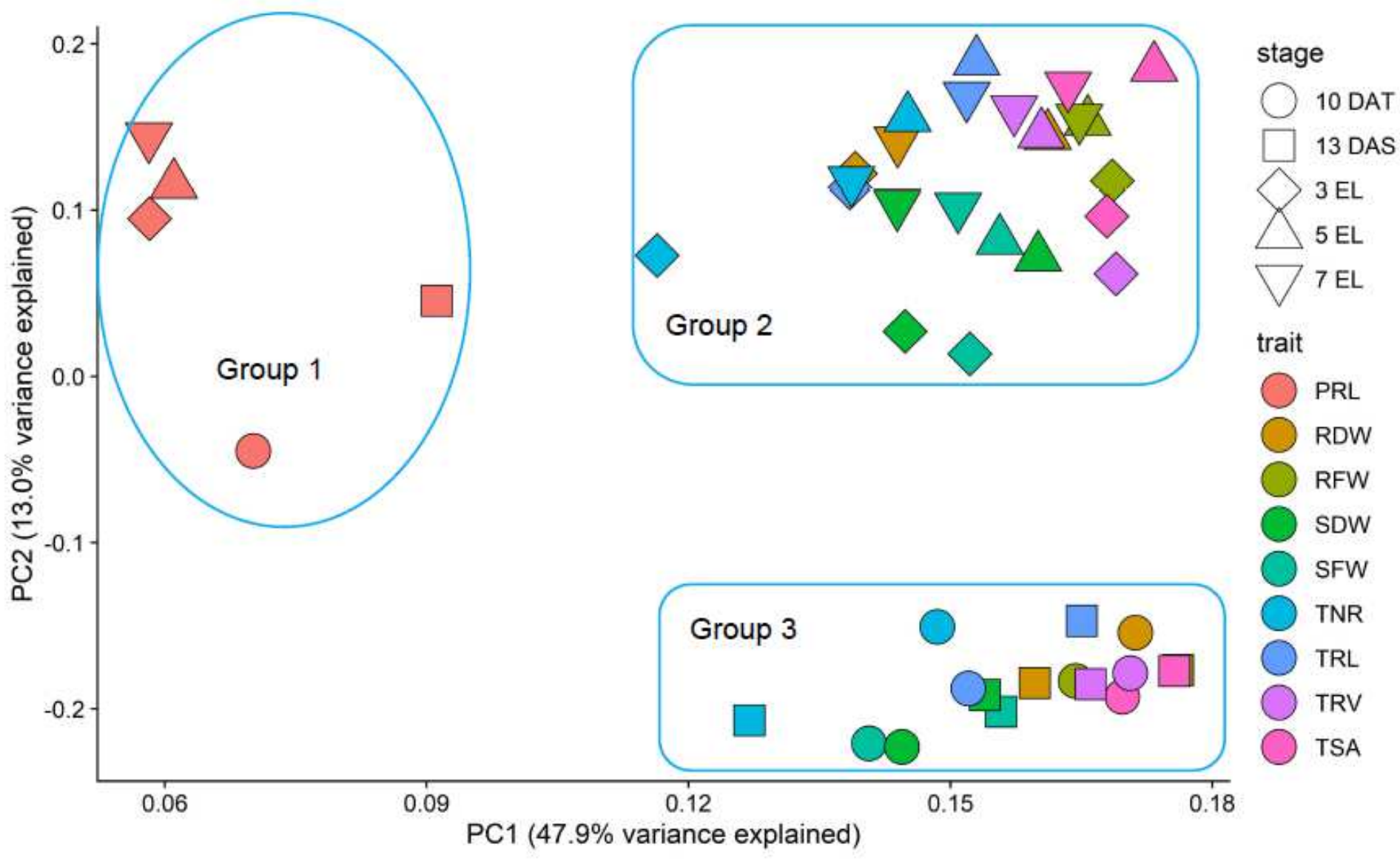

Figure 2

Principal component analysis (PCA) of captured traits at five stages in association population. Three obvious groups are indicated with blue circles. PRL trait was clustered into group 1. Other traits captured at 13 DAS and 10 DAT were clustered into group 3. The traits captured at $3 \mathrm{EL}, 5 \mathrm{EL}$, and $7 \mathrm{EL}$ were clustered into group 2. 


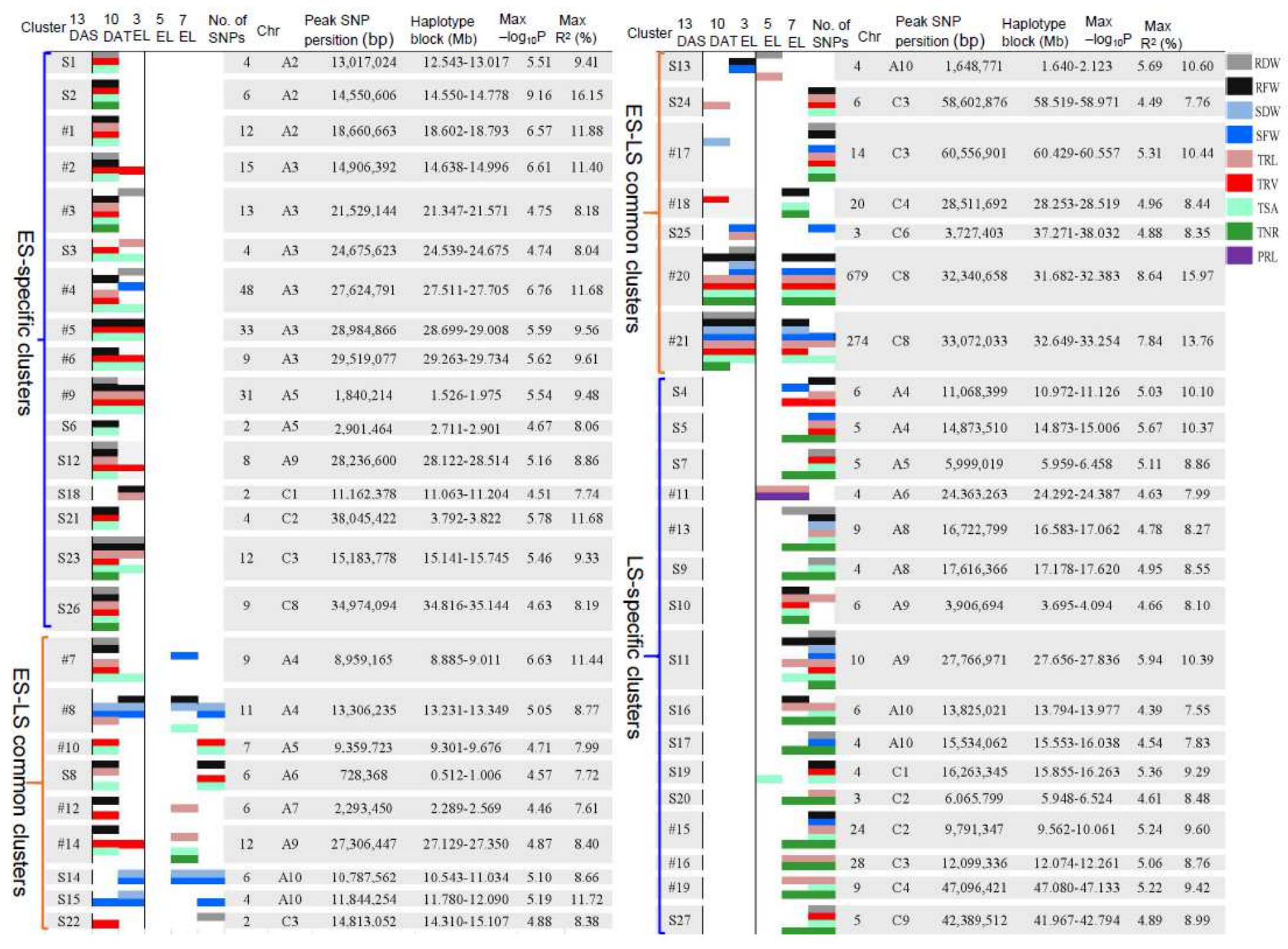

Figure 3

Genetic landscapes of QTL clusters obtained in this study. QTL clusters refer to significant SNPs with close proximity (within $1 \mathrm{Mb}$ ) and an LD of $\mathrm{r} 2>0.2$. Position, physical range, number, largest genetic variation explanation of significant SNPs of each cluster. \# represents clusters with multiple SNPs. S indicates the clusters harbouring single SNPs. ES-specific clusters: early stage specific clusters, including 13 DAS and 10 DAT; LS-specific clusters: late stage specific clusters, including 3 EL, 5 EL, and 7 EL; ES-LS common clusters: early and late stage clusters, including 13 DAS, 10 DAT, 3 EL, 5 EL, and 7 EL. 


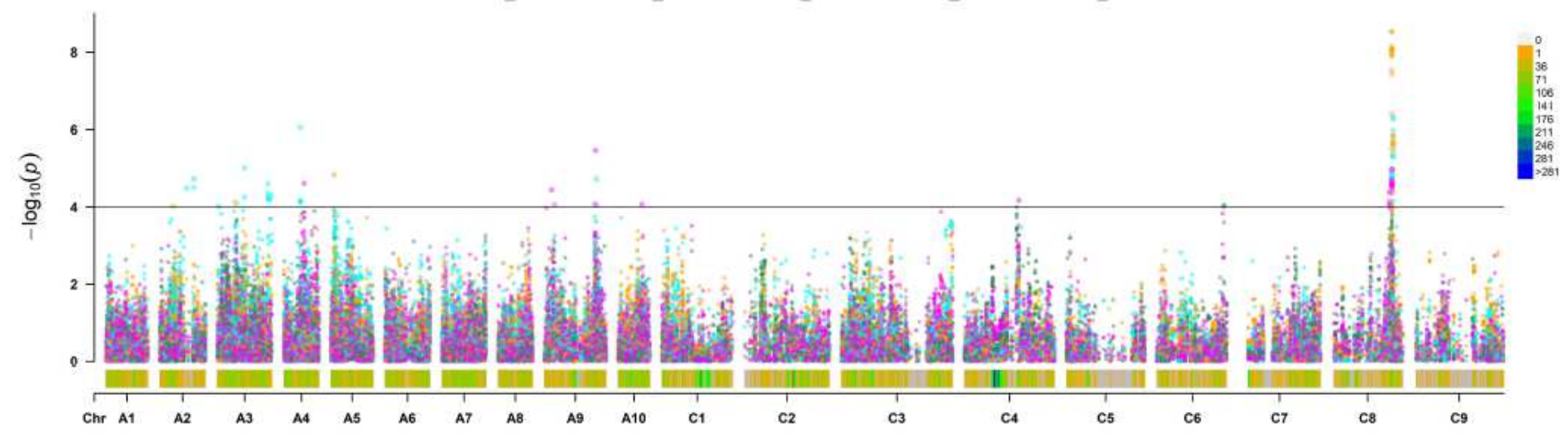

\section{Figure 4}

Manhattan plot of total surface area (TSA) at five stages. The different colours represent the TSA traitrelated SNPs at 13 DAS, 10 DAT, $3 \mathrm{EL}, 5 \mathrm{EL}$, and $7 \mathrm{EL}$, respectively. The horizontal black lines indicate the significance threshold of GWAS (- $\log 101 / 23,542=4.37)$. The $x$ axis shows the 19 chromosomes $(A 1-$ A10 and C1-C9) in B.napus. Each chromosome is scaled by the physical chromosome length.

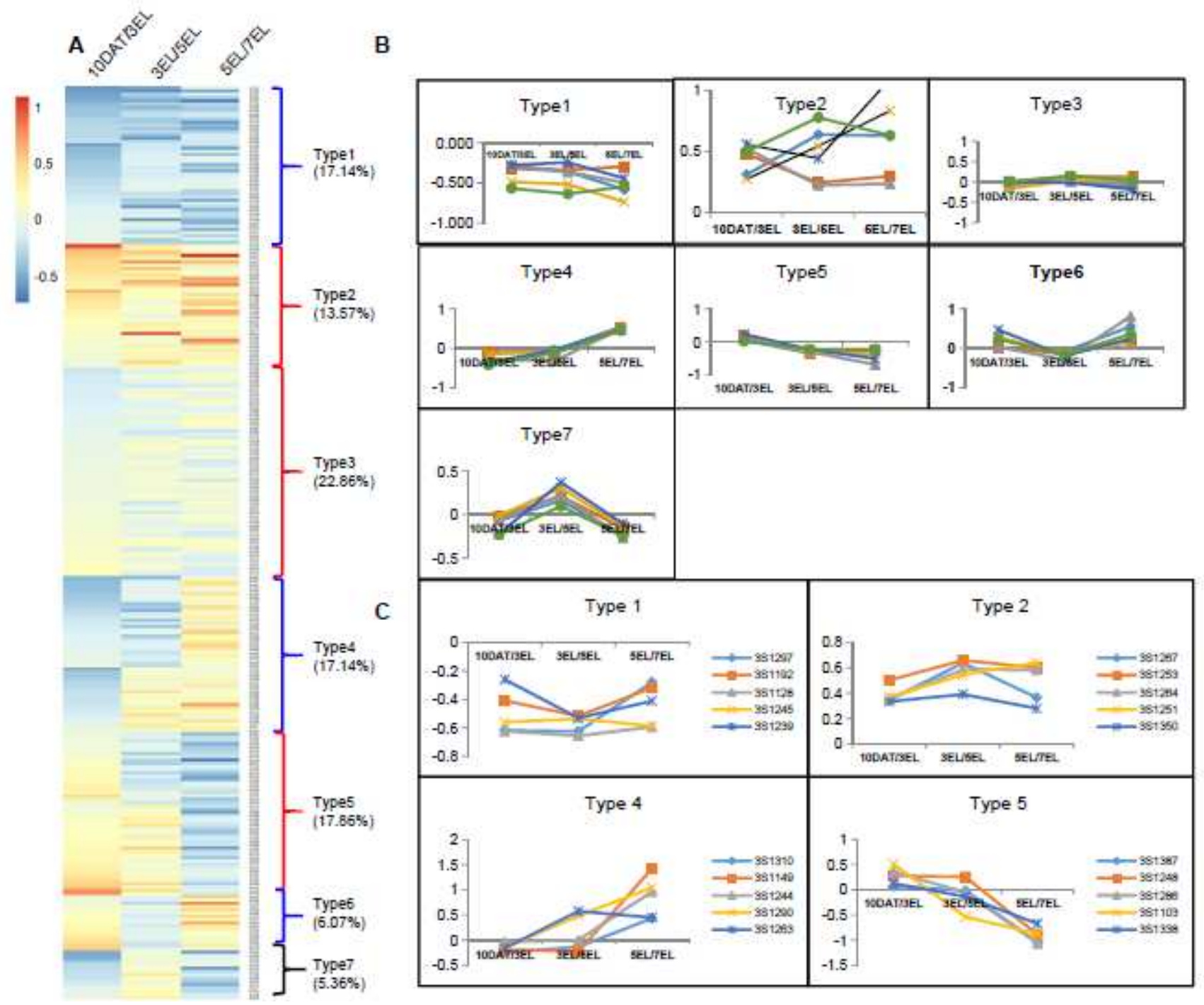




\section{Figure 5}

Clustering analyses of 280 accessions. (A) Hierarchical heatmap of 7 dynamic growth types of 280 accessions based on normalized GR values calculated by RFW. Red represents the GRs above the average, and green indicates the GRs below the average. (B) Diagram of 7 growth types with six accessions in each growth type. (C) Diagram of 4 transcriptome-sequenced growth types (type 1, 2, 4, and 5) with 5 accessions in each type.

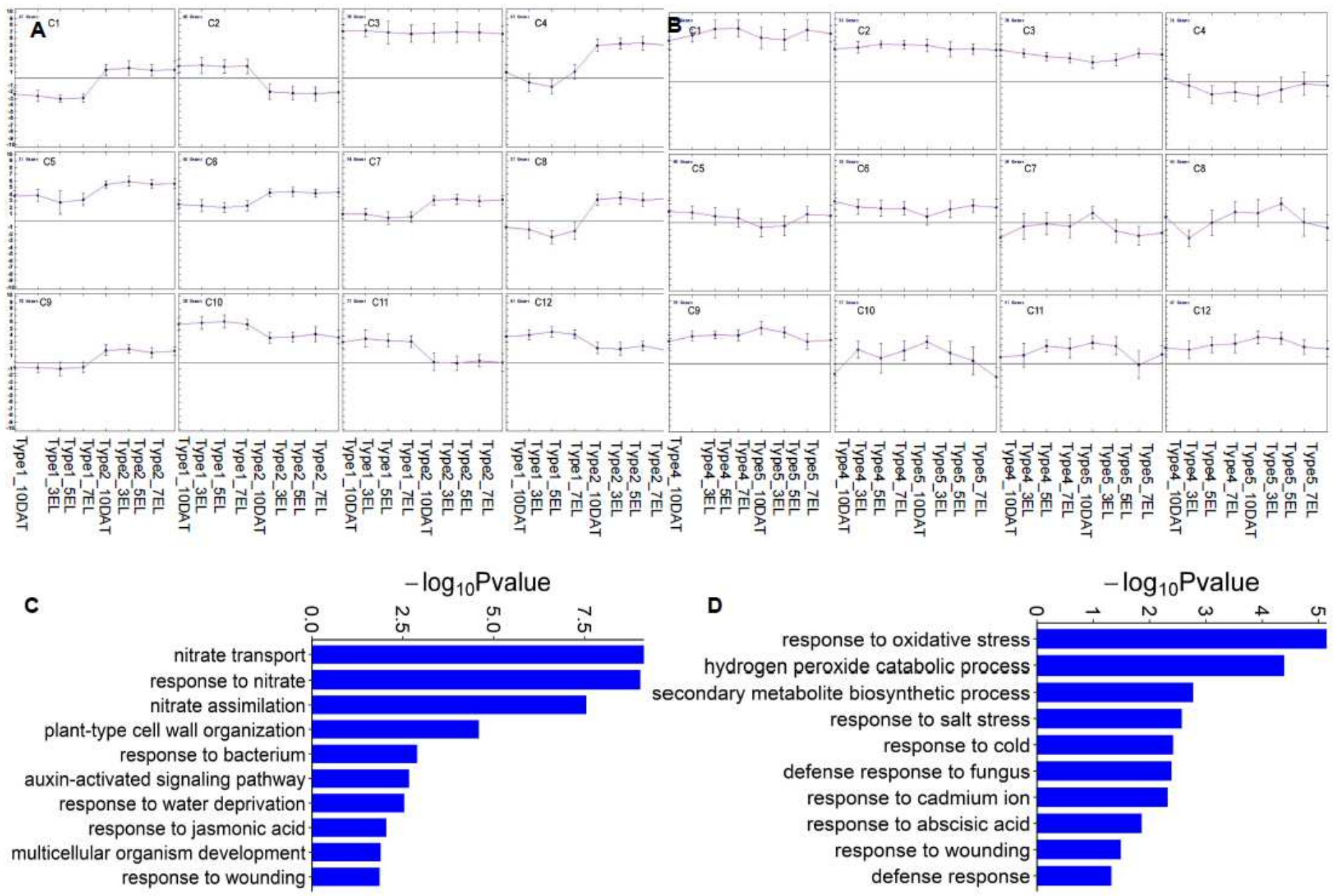

\section{Figure 6}

Information of persistent and stage-specific DEGs during root development in rapeseed. (A) K-mean clustering analysis of persistent DEGs in type 1 and type 2 . The $x$ axis indicates the samples at the four stages of type 1 and type 2 . The $y$ axis denotes the log2(FPKM). error bars represent the min and max data. (B) K-mean clustering analysis of the stage-specific expression genes in type 4 and type 5 . The $x$ axis indicates the samples at the four stages of type 4 and type 5 , and the $y$ axis denotes the log2(FPKM). error bars represent the min and max data. (C) GO terms enriched with the persistent DEGs in type 1 and type 2. (D) GO terms enriched with the stage-specific expression genes in type 4 and type 5 . 
A

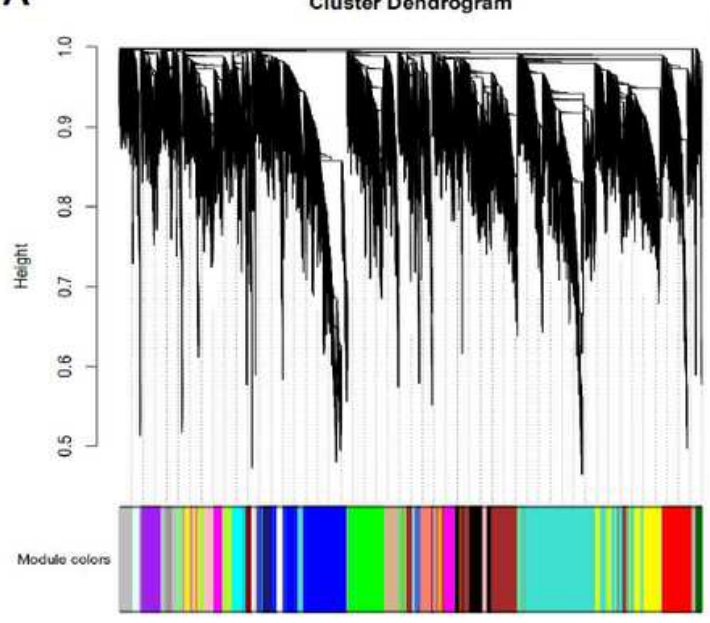

B

Edarkturquoise MEmagenta MEyellow MEdarkorange MEgreenyellow MEwhite MEcyan MEdarkred MEdarkgrey MEroyalblue MEskyblue MEbrown MEsalmon MEdarkgreen MElightgreen MEblack MEpink

MEturquoise MEgreen MEtan

MElightcyan MEpurple AEsaddlebrown MEblue

MEorange MEgrey60 MElightyellow AEmidnightblue MEred MEgrey
Module-sample relationships

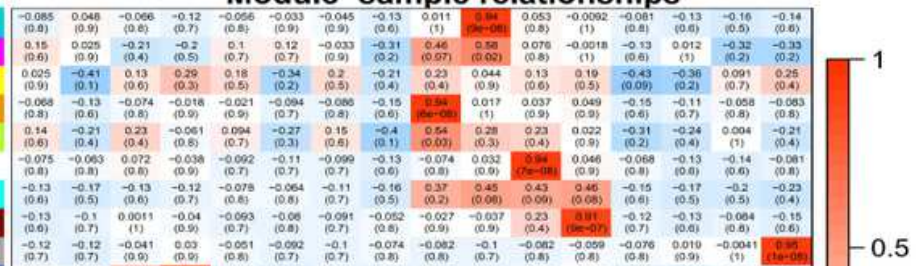

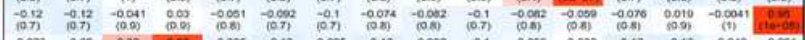

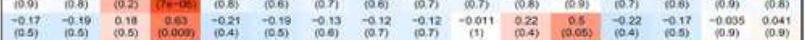

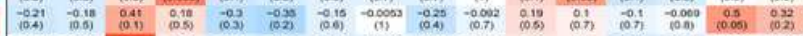

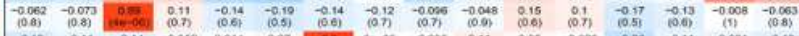

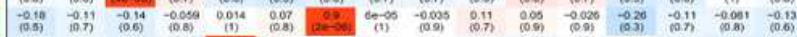

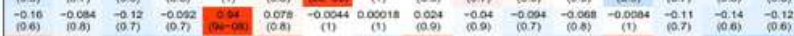

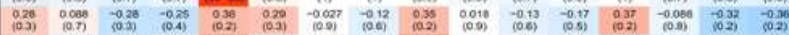

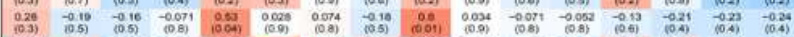

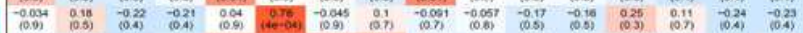

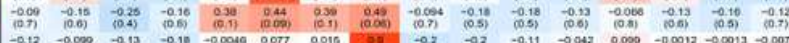

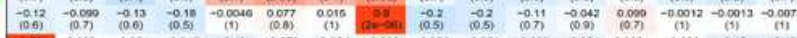

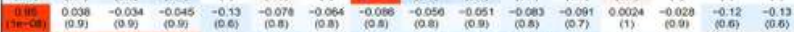

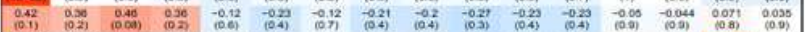

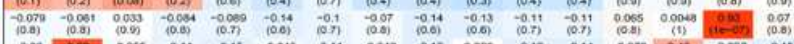

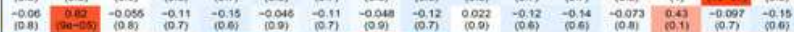

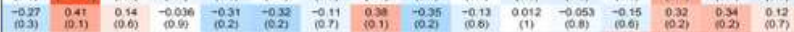

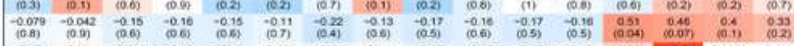

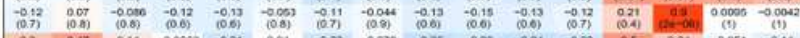

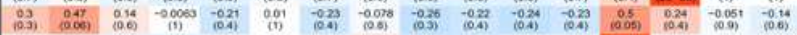

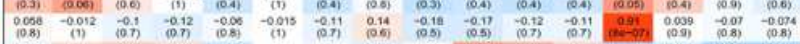
$\perp_{-1}$

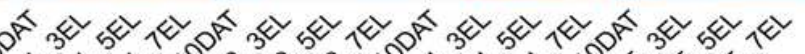
6.

\section{Figure 7}

WGCNA of gene expression matrix in rapeseed. (A) Hierarchical clustering tree (dendrogram) of genes based on co-expression network analysis. (B) Module-sample association. Each row corresponds to a module labeled with a color identical to that in $(A)$, and each column corresponds to a sample. 


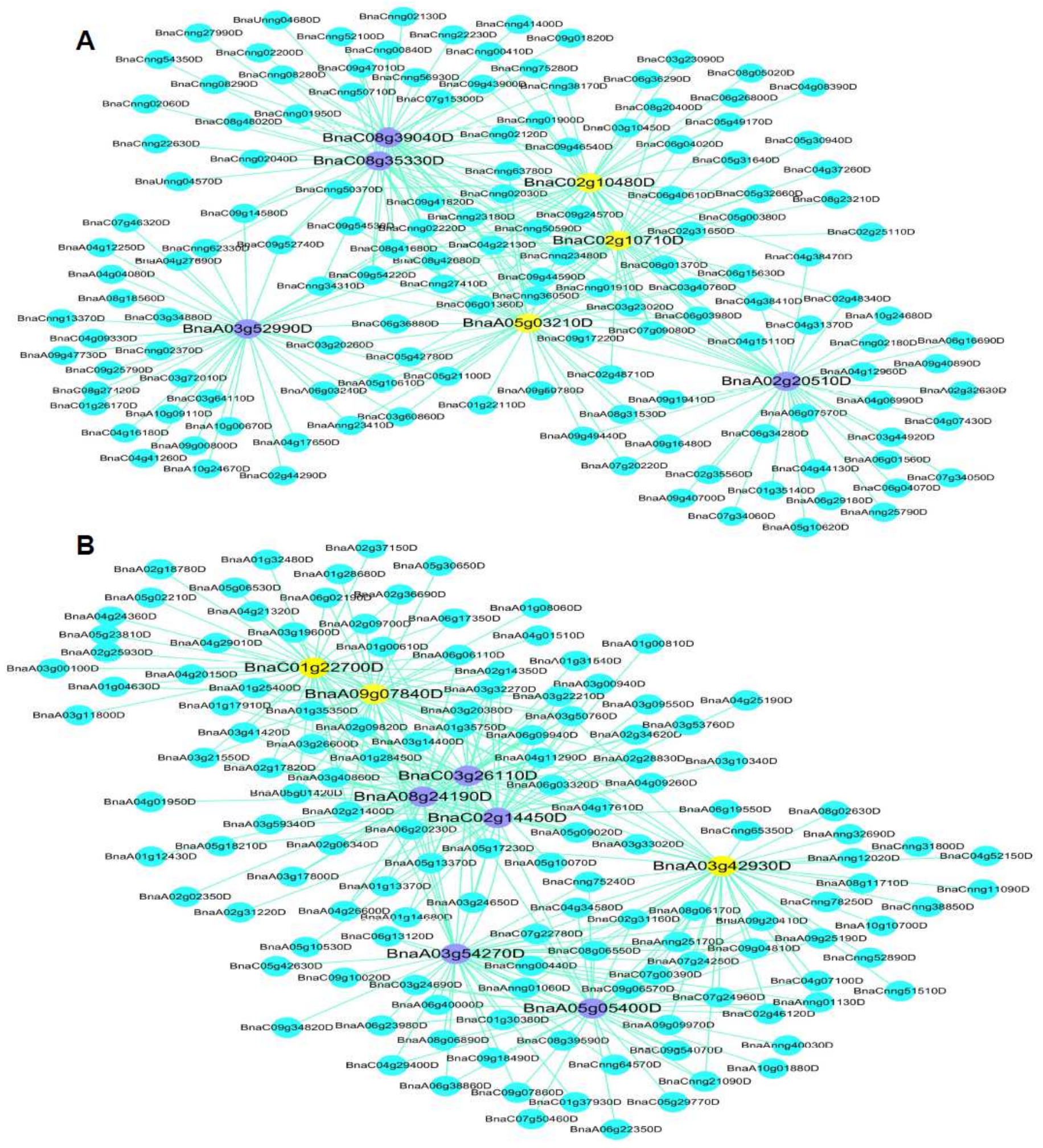

\section{Figure 8}

Networks of genes in green and red modules. (A-B) Correlation networks in green and red modules, respectively. Yellow colour in the network indicates the candidate genes overlapped by GWAS, WGCNA and differential expression analysis, and the purple colour in the network indicates the candidate genes overlapped by GWAS and WGCNA. 


\section{Supplementary Files}

This is a list of supplementary files associated with this preprint. Click to download.

- Supplementalfigures.pdf

- supplementarytables.xlsx 